Canadian University Music Review

Canadian University Music Review

Revue de musique des universités canadiennes

\title{
À propos de l'ouvrage de Lerdahl et Jackendoff : « A Generative Theory of Tonal Music "
}

\section{Célestin Deliège}

Numéro 4, 1983

URI : https://id.erudit.org/iderudit/1013901ar

DOI : https://doi.org/10.7202/1013901ar

Aller au sommaire du numéro

Éditeur(s)

Canadian University Music Society / Société de musique des universités canadiennes

ISSN

0710-0353 (imprimé)

2291-2436 (numérique)

Découvrir la revue

Citer cet article

Deliège, C. (1983). À propos de l'ouvrage de Lerdahl et Jackendoff : « A

Generative Theory of Tonal Music ». Canadian University Music Review / Revue de musique des universités canadiennes, (4), 141-183.

https://doi.org/10.7202/1013901ar

(c) Canadian University Music Society / Société de musique des universités canadiennes, 1983
Ce document est protégé par la loi sur le droit d'auteur. L'utilisation des services d'Érudit (y compris la reproduction) est assujettie à sa politique d'utilisation que vous pouvez consulter en ligne.

https://apropos.erudit.org/fr/usagers/politique-dutilisation/ 


\section{À PROPOS DE L'OUVRAGE DE LERDAHL ET JACKENDOFF: "A GENERATIVE THEORY OF TONAL MUSIC »}

\section{Célestin Deliège}

Cet ouvrage qui vient de paraître (1983), on le savait en préparation depuis huit ans. Ses auteurs étaient connus en France depuis que pendant près d'une journée de mai 1975, ils exposèrent les fondements de leur théorie (développés aujourd'hui dans les chapitres V à IX du livre) dans le cadre du colloque Linguistique-Musique que Nicolas Ruwet avait organisé à l'IRCAM. ${ }^{1}$ La rencontre entre les auteurs tient assez du hasard hegelien auquel croyait tellement André Breton: Fred Lerdahl, un compositeur new yorkais, mais aussi préoccupé de problèmes de logique, à qui l'évolution récente de la linguistique n'était pas étrangère. Ray Jackendoff, un linguiste dont les travaux faisaient déjà autorité, et qui appartient à cette frange de générativistes avant tout soucieux de ne rien énoncer qui n'ait été vérifié en psychologie; mais en outre, un bon musicien exécutant. Les deux hommes se rencontrent lors d'un séminaire organisé au M.I.T. fin 1974 par Irving Singer et David Epstein (Préface). ${ }^{2}$

L'ouvrage pourrait bien être le plus important en matière de grammaire musicale écrit depuis Der freie Satz (Schenker 1935). Comme ce fut le cas pour ce dernier ouvrage, mais plus rapidement sans doute et à une plus grande échelle, celui de LerdahlJackendoff semble capable d'opérer une reconversion des mentalités dans l'approche de la musique en général et d'offrir aux études musicologiques une chance de rénovation.

\section{Chapitre I}

Le titre Théorie générative ne doit pas être lu comme une tentative de transfert, vers la musique, de la théorie standard de 
Chomsky portant sur les langues naturelles. Le linguiste Jackendoff était suffisamment averti pour savoir d'où pouvait venir le mal: quelques tentatives précédentes avaient indiqué l'impasse. Le but est ici de décrire, par un système de règles, l'intuition d'un "auditeur expérimenté". Nous sommes donc en présence d'un travail de prospection fondé sur des données de la psychologie cognitive. La linguistique générative n'interviendra qu'en un arrière-plan méthodologique.

Quant à la seconde partie du titre, Musique tonale, il faut l'entendre avec une restriction significative; délibérément, les auteurs n'étudient que des processus monophoniques.

Dans l'état actuel du développement de la théorie, nous traitons toute musique comme essentiellement homophonique, c'est-àdire que nous n'assumons qu'une seule analyse de groupement pour toutes les voix d'une pièce. Pour les variétés plus contrapuntiques de musique tonale où cette condition n'est pas remplie, notre théorie est inadéquate. Nous considérons qu'une extension de notre théorie, englobant la musique polyphonique, est d'une grande importance, toutefois nous n'en traiterons ici que par approximation (p. 37). ${ }^{3}$

Pour les auteurs,

... une oeuvre musicale est une entité construite mentalement, dont les partitions et les exécutions sont des représentations partielles par lesquelles l'oeuvre est transmise.... La tâche centrale de la théorie musicale devrait être d'expliquer cette organisation produite mentalement. Vue de cette façon, la théorie prend place dans les domaines de la psychologie cognitive comme les théories de la vision et du langage (p. 2). ${ }^{4}$

La notion d'auditeur expérimenté risque toutefois de faire problème. Qui est-il? ... Est-ce un personnage de conte de fée? Est-ce le "bon auditeur» d'Adorno (1962: chap. I)? Les auteurs mesurent la difficulté et reconnaissent que c'est une idéalisation. Leur définition dissipe pourtant tout malentendu: il ne s'agit pas

... de la conscience exacte qui saisit les structures musicales; un auditeur acculturé n'a jamais besoin d'avoir étudié la musique. Nous nous référons plutôt à la connaissance largement inconsciente (l' «intuition musicale») dont l'auditeur entoure son audition - une connaissance qui le rend capable d'organiser et de découvrir la cohérence des patterns de surface: hauteur, attaque, durée, intensité, timbre, etc. Un tel auditeur est apte à identifier une oeuvre que, précédemment, il ne connaissait pas comme exemple relevant d'un idiome déterminé; de reconnaître 
les éléments d'une pièce comme typiques ou anomaux; d'identifier une erreur d'exécution comme pouvant produire une configuration "agrammaticale "; de reconnaître diverses espèces de répétitions et de variations structurales; et, généralement, de saisir l'oeuvre à l'intérieur de son propre langage (p. 3). ${ }^{5}$

Pour qu'une théorie puisse être déduite, cette première idéalisation doit être complétée par une seconde: l'auditeur expérimenté est sensé tout entendre:

Les deux idéalisations que nous avons adoptées, celle de l'auditeur expérimenté et celle de l'état final de sa compréhension sont comparables à des idéalisations faites ailleurs en psychologie cognitive. Sans quelque simplification le phénomène visé par l'enquête scientifique s'est presque toujours révélé réfractaire à l'investigation rationnelle (p. 4). ${ }^{6}$

Mais

...il serait sans intérêt de théoriser sur le processus mental, avant d'entreprendre l'étude de l'organisation à laquelle le processus conduit. Ceci n'est qu'un choix méthodologique de notre part (ibid.). ${ }^{7}$

La reconstitution d'une intuition doit en effet être appuyée préalablement sur une donnée structurée: soit une théorie bien constituée.

Une théorie formelle de la musique rendra possible des hypothèses substantielles sur ces aspects de la compréhension musicale qui sont innés. Les aspects innés se révèleront euxmêmes comme des «principes universaux » de la grammaire musicale (ibid.). ${ }^{8}$

Dès lors, n'y a-t-il pas une contradiction implicite entre le fait de se référer aux capacités génératives innées et le fait de restreindre la théorie à la structure de la musique tonale? Les auteurs ne le pensent pas. Ils ont d'ailleurs le regard tourné vers d'autres grammaires, mais ils ont besoin d'un système cohérent et bien éprouvé pour établir une formalisation qu'ils conçoivent scientifiquement comme hypothèse vérifiable et falsifiable. Le type de formalisation choisi demeurera très concret:

La formalisation envisagée pour elle-même est pour nous dépourvue d'intérêt; elle ne devient intéressante que pour autant qu'elle serve à exprimer des généralisations musicales et psychologiques, et à produire des résultats empiriques plus précis (p. 5). ${ }^{9}$ 
Aucune théorie autre que la théorie tonale n'aurait pu satisfaire aux besoins ici visés. Lerdahl et Jackendoff sont conscients de ne pouvoir prendre en compte la totalité des intuitions de l'auditeur, et ils se sont volontairement limités à cette part de l'intuition capable d'appréhender les hiérarchies (p. 8). Or de tous les systèmes musicaux connus, ce n'est pas s'aventurer que de dire que le système tonal, et les formes qui en sont dérivées, sont les plus fortement et les plus rationnellement hiérarchisés. Ainsi orientée, la recherche va s'attaquer à quatre composants de base: 1) la structure des groupes, 2) la structure métrique, 3) la réduction des trames temporelles (time-span reduction), 4) la réduction des prolongations (prolongational reduction). ${ }^{10}$

Ces différents types de structures hiérarchiques sont essentiellement contrôlés par deux catégories de règles, celles de bonne formation, connues aussi en syntaxe et en sémantique du langage ordinaire; et les règles préférentielles, lesquelles sont une émanation de la Gestaltpsychologie et n'ont aucun équivalent dans la théorie linguistique. Les règles de bonne formation sont des règles de base; les règles préférentielles sont déterminées par les choix intuitifs de l'auditeur. "La description d'une structure préférée tendra à établir un rapport entre des intuitions disparates et révèlera des patterns structuraux réguliers " (p. 9). ${ }^{11}$ L'input de ce système théorique est toujours la structure de surface, autrement dit la séquence réelle; l'output est tracé par le parcours de l'intuition de l'auditeur à travers les règles préférentielles (p. 11).12

\section{Chapitres II à IV}

L'intuition de l'auditeur est d'abord reconstituée à partir de l'aspect rythmique. Mais comment établir, en musique tonale tout au moins, une théorie du rythme sans la fonder sur une bonne lecture des rapports de hauteurs? C'est naturellement ce que les auteurs ont compris d'emblée. Ils vouent, toutefois, une confiance assez grande au facteur métrique. Bien qu'ils reconnaissent que la grille métrique est "comme un papier peint" (p. 28), et que cette structure perd rapidement de son efficacité au fur et à mesure que l'on s'élève dans les niveaux de l'analyse (p. 21), ils la traitent indépendamment des groupes, sans ignorer, bien sûr, la puissante interaction existant entre mètres et 
groupes pour fonder le rythme. Le lecteur vivra d'ailleurs pleinement l'interaction dans la réduction des trames temporelles. Quoi qu'il en soit, on peut s'étonner de trouver dans la théorie une place aussi considérable accordée au mètre; car enfin, le mètre en musique tonale n'a jamais été plus qu'un étalon, et cette structure n'a jamais été acceptée par les comıpositeurs que pour mieux être transgressée. La question est naturellement de savoir si cette transgression même n'a pas constitué la reconnaissance la plus éclatante de l'importance du phénomène. Pour l'instant, je répondrai à cette interrogation que la structure métrique est prégnante dans la mesure où elle s'impose ellemême comme cadre rythmique. Ainsi trouve-t-elle son maximum d'efficacité dans les pièces composées en référence à la danse et son minimum d'intérêt dans les pièces de style fugué. Entre les deux pôles, s'insère une série de degrés d'efficacité où vont se trouver des cas d'espèces. En dépit de la différence profonde existant entre poésie et musique, que je n'essaierai pas de minimiser, il me semble que la récente théorie de l'alexandrin de Benoit de Cornulier est éclairante, même pour le musicien. En gros, ce poéticien distingue en s'appuyant, comme Lerdahl et Jackendoff, sur la capacité générative du sujet, entre une appréhension synthétique du vers référée à sa structure métrique répartie entre les hémistiches, et une perception analytique appuyée sur les variantes rythmiques qui peuvent apparaître à l'intérieur des hémistiches ou les débordements dans l'enjambement (Cornulier 1982). Ne pouvons-nous estimer, de manière équivalente, que la grille métrique serait le cadre synthétique sous-jacent de notre audition, alors que le rythme concret, tel qu'il se lit dans la structure des groupes et se laisse réduire dans les divers niveaux de la structure temporelle, serait l'écoute analytique? En acceptant simultanément ces deux conditions de la perception de la durée, nous rejoignons avec moins d'embarras, me semble-t-il, une structure métrique toujours assez neutre, élément régulateur de l'audition. Des réserves très marquées devront toutefois être maintenues dans le cas de pièces très contrapuntiques; mais la théorie de nos auteurs est limitée aux structures monophoniques: ce point ne doit pas être perdu de vue.

L'auditeur infère un pattern métrique régulier à partir de l'accent métrique qu'il dégage de la complexité rythmique, en dépit des conflits auxquels on vient de faire référence, et qui très souvent résultent de la syncopation (pp. 17-18). Le pattern éta- 
blit une périodicité hiérarchique dont l'élément minimal est la battue, repère idéal dépourvu de durée. Le pattern métrique est figuré par les auteurs de la manière suivante (p. 20):

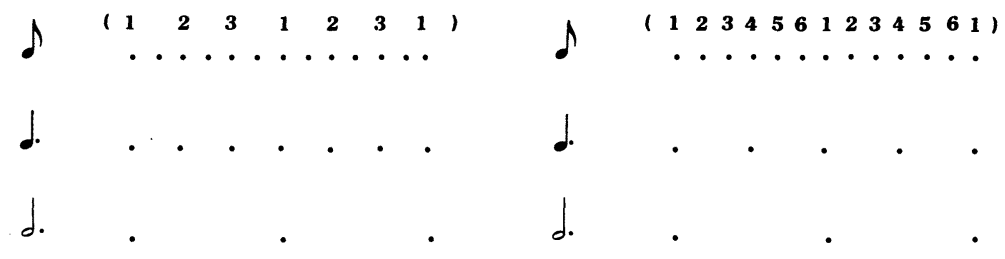

Exemple 1

Cet exemple, il est vrai, montre un niveau de division de la battue (la croche), mais on aura compris que la battue reste néanmoins l'élément impliquant. Au-delà de l'alternance des temps forts et faibles, on notera que la théorie prévoit une alternance de mesures fortes et faibles, ce que les structures musicales, il faut le reconnaître, ne corroborent que moyennant des adaptations parfois subtiles. En fait, si alternance de mesures fortes et faibles il y a, c'est bien toute l'organisation de la surface musicale qui peut l'engendrer, il ne faut pas perdre de vue que l'alternance des temps forts et faibles, dans une même mesure, est déjà souvent une vue assez abstraite. Bien que Lerdahl et Jackendoff se montrent clairement conscients de la faible portée de la perception du mètre sur une grande échelle (pp. 21 à 25), il me paraît que, sur ce point, ils restent trop conciliants et défendent des représentations assez idéales.

Manifestement, dans la reconstitution de l'écoute intuitive, de mon point de vue, la structure métrique ne peut être qu'un appoint très sous-jacent. Ce qui le plus importe, c'est la capacité de l'auditeur de grouper les unités, et la manière de hiérarchiser les groupes. "Les deux sont nécessaires " précisent les auteurs (p. 29) et ils n'entendent pas que les propriétés des deux types d'organisation soient confondues: «Les groupes ne reçoivent pas d'accent métrique et les battues ne possèdent aucun groupe inhérent " (p. 26). ${ }^{13}$ Ainsi, ont-ils eu raison de bien distinguer préalablement (p. 17) entre trois espèces d'accents: l'accent phénoménal qui est en fait tout accent audible dans la structure de surface, notamment l'accent dynamique; l'accent métrique, marqué par le temps fort des mesures; et l'accent structural, que 
pour abréger je comparerai à notre très ancienne notion de thesis. Entre ces deux derniers types d'accent et la place qu'ils occupent, va se jouer essentiellement l'interaction entre structure de groupes et structure métrique. Cette relation s'exprime dans la structure des trames temporelles: selon qu'il y aura ou non coïncidence entre début de groupe et battue la plus forte au plan métrique, les deux structures seront dites en phase ou hors phase (pp. 29 et 30). Là se situe le point crucial d'appréhension de la structure rythmique. Par la discussion d'exemples appropriés, les auteurs montrent clairement comment l'accent métrique et l'accent structural agissent indépendemment pour la constituer. La théorie de l'accent structural les amène à développer une discussion de la prosodie des groupes, différente de celle de Cooper et Meyer (1960) qui réduisaient la prosodie tonale à la rythmique grecque, et faisaient simultanément supporter à la longue opposée à la brève, une valeur d'accent prosodique et métrique. L'accent structural apparaît ici comme centre de gravitation du groupe; on n'est pas très loin de la notion de groupe anacrouse-accent - désinence telle que l'entend Messiaen (1944: 49) et que j'ai cherché à systématiser, autant que faire se peut (Deliège 1979 et 1984: chap. V). Mais Lerdahl et Jackendoff ne semblent pas apercevoir la katalase en dehors de la cadence. Une théorie rythmique doit pouvoir cependant aller plus loin et comprendre l'occurrence de désinences extracadentielles et inclure des accents secondaires tels ceux que je dénomme accent arsique ou accent désinentiel. Selon qu'ils se trouvent «à gauche " ou "à droite » de l'accent principal.

Les groupes, comme cela a été dit plus haut, sont appréhendés par les règles de bonne formation et les règles préférentielles. La place manque pour les commenter, je les énumérerai simplement. Au reste, elles parlent d'elles-mêmes. Dans le but d'unifier les représentations, je conserverai les abréviations anglaises, soit GWFRs pour Grouping Well-formedness Rules, et GPRs pour Grouping Prefence Rules. ${ }^{14}$

Cinq GWFRs sont énoncées (pp. 37 à 39). GWFR 1 déclare que seules des suites d'événements contigus peuvent constituer un groupe. " La condition de contiguïté est ce qui rend particulièrement efficace la liaison dans la notation pour la représentation des intuitions de groupement " (p. 37). ${ }^{15}$ GWFR 2 rappelle que toute pièce constitue un groupe. GWFR 3 dit qu'un groupe peut contenir de plus petits groupes. GWFR 4 dit que si un groupe G1 contient des parties de G2, il doit contenir la totalité 
de G2. GWFR 5, enfin, stipule que si un groupe G1 contient un plus petit groupe $\mathrm{G} 2$, alors la totalité de G1 doit être subdivisée. En résumé, ces règles traduisent donc des contraintes d'organisation touchant essentiellement les solidarités et les hiérarchies.

Les GPRs, au nombre de sept, sélectionnent des choix "parmi un nombre d'analyses logiques possibles». Il s'agit donc d'une hypothèse qui propose l'analyse la plus adéquate ou la plus « stable » après que « toutes les règles préférentielles ont été prises en compte» (p. 42). Après que le caractère non arbiraire des règles ait été bien établi par des exemples basés sur la perception visuelle et la perception musicale élémentaires, elles sont énoncées en deux groupes (pp. 43 à 52). GPRs 1,2 et 3 concernent des détails de la structure locale. GPR 1 spécifie que la préférence s'écarte fortement des groupes constitués d'un seul événement et évite aussi les très petits groupes. GPR 2 est référée à la proximité: elles sont délimitées sur cette base a) par les liaisons et b) par les points d'attaque. GPR 3 est référée au changement: a) de registre, b) de dynamique, c) d'articulation, d) de longueur. GPRs 4, 5, 6 et 7 ont trait à l'organisation de groupes de niveaux plus élevés pouvant atteindre jusqu'aux structures globales. GPR 4 est une règle d'intensification à un niveau supérieur de GPRs 2 et 3 . GPR 5 contrôle la symétrie des groupes, et GPR 6, les parallélismes. GPR 7 vérifie la stabilité dans les trames temporelles et la prolongations: les deux dimensions fondamentales de la théorie donnant lieu à réduction.

Parmi ces règles, celle qui touche le parallélisme a bien été jusqu'ici le support le plus confortable des analyses. Je note à ce sujet une mise en garde des auteurs qui me paraît devoir être opportunément rappelée aux étudiants:

L'importance du parallélisme dans les structures musicales ne doit pas être surestimée. Plus on peut détecter de parallélisme, plus l'analyse gagne en cohérence interne et moins indépendante devient l'information organisée et retenue dans l'audition et le souvenir d'une pièce (p. 52$){ }^{16}$

De ce point de vue, j'ai toujours proposé comme contreexemple les oeuvres qui ne comportent aucun parallélisme ou en comportent très peu, et qui cependant appellent des analyses non moins pertinentes. Le parallélisme est d'ailleurs un gros problème structural qui dépasse de loin l'analyse musicale, c'est un problème de psychologie générale, Lerdahl et Jackendoff le rappellent opportunément (p. 53). 
Dans le contexte, les règles préférentielles entrent en concurrence: elles se renforcent mutuellement ou entrent en conflit. Comment décider des préférences dominantes? On a songé à pourvoir les règles d'une grille numérique, mais les auteurs ont renoncé à un tel projet, n'y voyant qu'une source d'arbitraire. Ils ont également renoncé à un formalisme mathématique ou "métamathématique " (Tarski) pour formuler les douze règles dont il vient d'être question et les autres, préférant le langage ordinaire. Ayant le sentiment d'aller a contrario par rapport aux tendances de l'époque, ils se défendent sur ce point; mais je crois que tout lecteur conscient du mauvais mariage qui ne pourrait que résulter d'une alliance entre théorie musicale et dogmatisme, les suivra sans la moindre peine. ${ }^{17}$

Les conflits de règles préférentielles posent naturellement plus de problèmes que leur concordance. Une question importante et difficile est, par exemple, de déterminer la valeur prosodique d'un son pivot: est-il désinence dans le groupe actuel ou anacrouse du groupe suivant? Je tenterai d'articuler une brève discussion de cette question posée ici même (p. 63) à propos du début de la Sonate, K. 331, de Mozart et du début de l'Intermezzo, Op. 119, No 3, de Brahms (Nattiez 1975: 292 et sq.): les deux cas présentent une analogie incontestable. Cette brève discussion devrait mettre en évidence la nécessité de compléter les règles préférentielles de la perception par une série d'autres touchant l'élaboration, quand il s'agit de messages individuels de source savante. Nous plaçons en regard les deux exemples (cf. ex. 2 et 3).

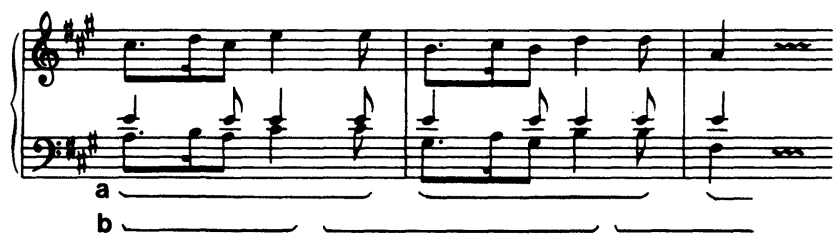

Exemple 2 


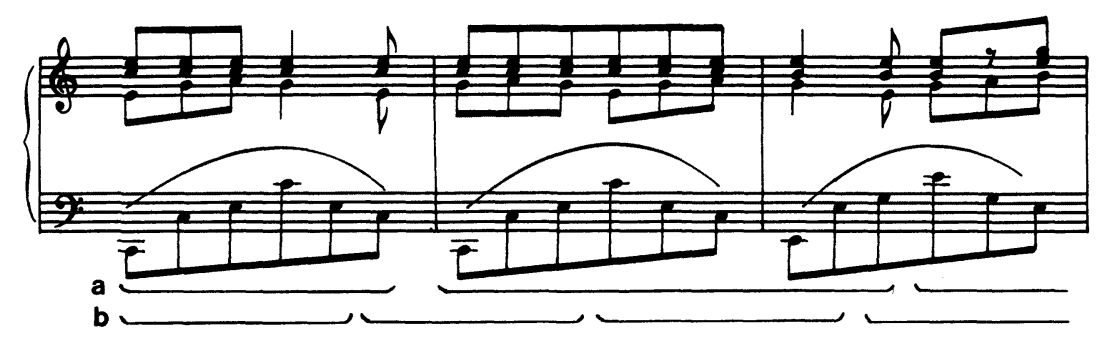

Exemple 3

Chacun comporte deux interprétations a et b. Le cas Mozart a été étudié par Leonard Meyer (1973) à qui Lerdahl-Jackendoff renvoient pour apprendre que l'interprétation $b$ a eu aussi ses avocats. Heureusement, en se reportant à la référence en question, on constate que Meyer récuse sans appel l'interprétation b. Le problème a été bien cerné par Meyer (1973: 29-40). Nous apprenons que l'interprétation a serait le phrasé de Mozart mis en évidence par Nathan Brother, et que l'interprétation best le fruit de l'édition Peters établie par Kohler et Ruthardt. Quant au cas Brahms, Nattiez nous informe que l'interprétation a est celle de Julius Katchen, et l'interprétation $b$ celle de Walter Klein. Lerdahl et Jackendoff préfèrent l'interprétation a de Mozart, mais ils ne récusent pas, pour autant, l'interprétation b: elle s'accorde avec la configuration de certaines variations, mais surtout les règles préférentielles de perception obligent; et comme il est difficile d'en départager la puissance par une évaluation à priori, il faut accepter le libre jeu concurrentiel des règles en question. Nattiez, lui aussi, reste neutre devant les deux interprétations de Brahms: il applique la méthode analytique élaborée par Ruwet (1972: chap. 4) favorisant la répétition des séquences. L'interprétation a découle d'un découpage de niveau supérieur, l'interprétation $b$, d'un découpage de niveau plus petit. Englobant les deux exemples dans un même raisonnement permis par leurs points d'analogie, je résumerai les faits en disant simplement: aux endroits du litige les interprétations a incluent la désinence et excluent l'anacrouse; les interprétations $b$, au contraire, favorisent l'anacrouse et rejettent la désinence. Mais pas plus que Leonard Meyer, je ne resterai neutre: je déclare formellement que les interprétations $b$ sont incorrectes, voire totalement aberrantes. Nous touchons là un point limite 
des règles préférentielles de perception. On pourrait par exemple, formuler une sorte d'anti-règle préférentielle déclarant: sous certaines conditions les règles préférentielles de la perception permettent au virtuose de conforter des traditions de la « haute virtuosité » en préférant l'aberration. Mais comment en sortir? Car on l'aura compris, du moins je l'espère, il ne s'agit pas du tout de revenir sur l'acquis que constituent les règles préférentielles. De fait, mon point de vue est assez simple, et partant du langage parlé, je l'esquisserai ainsi: les règles préférentielles sont fondées sur l'intuition, elle-même dérivée de facultés innées; le sujet mis en contact avec un environnement qui est pour lui indicateur de bonne formation, organise son langage sur la base des préférences de son intuition constamment réordonnée et stimulée par les normes de bonne formation. Cette réorganisation constante des préférences au contact de la norme a lieu probablement de manière largement inconsciente. Cette beaucoup trop sommaire description du processus d'acquisition de la langue n'est sans doute rien de plus qu'une mise en relation de la performance et de la compétence: mots que l'on n'ose plus prononcer aujourd'hui tant la circonspection semble avoir manqué au niveau du traitement de ces notions. Mais les objets de civilisation, comme les appelait Francastel, le poème clos, le message musical non moins clos, peuvent-ils vraiment être traités comme la langue? Je puis tenter, il est vrai, et avec le succès de la théorie qui nous occupe, une théorie générative de la tonalité en général, une théorie du système fonctionnant hiérarchiquement et par le jeu de certains mécanismes; mais quand je passe à la théorie de l'oeuvre, à la grammaire de Mozart, de telle oeuvre de Brahms, de Baudelaire, de Mallarmé et de qui que ce soit, j'ai probablement besoin d'une nouvelle série de règles supplémentaires de bonne formation surtout, et de règles préférentielles d'élaboration touchant les préférences perceptives qui ont organisé l'oeuvre que j'aborde. Moyennant cette attention indispensable, avec Nattiez et Molino, je lève mon verre au souvenir de Valéry qui nous plaçait alternativement du côté du poïétique et de l'esthésique. Manquant de place pour m'étendre sur cette importante donnée, pour l'instant, je me bornerai à relever les faits les plus simples qui doivent permettre de réfuter les interprétations $b$ dans les exemples qui nous occupent. Ce sont, en fait, des conséquences esthésiques du poïétique: ce qui indique, en outre, la complémentarité dialectique qui réunit ces deux pôles. Je ne reprendrai pas ici l'argumentation de Leonard Meyer par manque de place et 
aussi parce que je n'assume pas entièrement la théorie prosodique de Cooper et Meyer qui sert ici partiellement de mode de défense; mais encore parce que le mode de réfutation fait intervenir des conditions qui, transformant le contexte, auraient permis l'interprétation $b$; je renvoie toutefois le lecteur intéressé à l'argumentation de Meyer, intéressante, et pertinente à l'intérieur de sa théorie. Les raisons que j'invoquerai, ici, sont à la fois extrêmement simples et générales. Dans les deux cas, elles font principalement appel au mètre et au rythme de l'harmonie.

1) MOZART. Une analyse schenkerienne bien comprise, c'est-à-dire, comme je le souhaite (Deliège 1982 et 1984), pouvant comprendre plus d'une ligne génératrice (Urlinie) surmontant la série des fondamentales, doit nous aider à déduire le rythme de l'harmonie et ce rythme à son tour permet de dégager la définition prosodique adéquate. Les exemples 4 et 5 [cf. page suivante] recoupent largement l'analyse de Schenker rappelée par Lerdahl \& Jackendoff (p. 276):

Le niveau a recoupe celui de l'Ursatz, niveau que généralement je ne pratique pas, mais qui ici trouve son application du fait de la brièveté de la séquence, et de la nécessité de maintenir la dominante au niveau supérieur de manière à rejoindre celle de la mesure 5. Il corrobore la réduction de la trame temporelle de Lerdahl-Jackendoff. Le niveau $b$ rejoint (mais c'est un hasard) celui du Mittelgrund schenkerien et l'analyse de Schenker. Il rejoint aussi la réduction de prolongation des auteurs; il offre déjà la possibilité de dégager l'accent et la désinence de premier niveau sur base de la règle prosodique que j'ai dénommée règle A.T.K. qui décrit la partition arsique-thétique-katalectique. Le niveau $c$ est un équivalent du Vordergrund: il recourt à la technique du déploiement (Ausfaltung) pour décrire la conduite de voix, il trace correctement le dessin contrapuntique et le rythme de l'harmonie au niveau inférieur. Il corrobore la structure normative exigée par le composant de prolongation dans la théorie de Lerdahl-Jackendoff (cf. infra).

L'exemple 5 montre l'application de la règle A.T.K. aux niveaux $b$ et $c$. Toute autre application de cette règle favorisant l'interprétation $b$ de l'exemple 2 violerait cette analyse fondée sur la description du contexte dans l'exemple 4. Or, aucune description qui s'en écarterait délibérément ne pourrait en restituer l'évolution par rapport à l'ensemble des paramètres qui le constituent. ${ }^{18}$ 

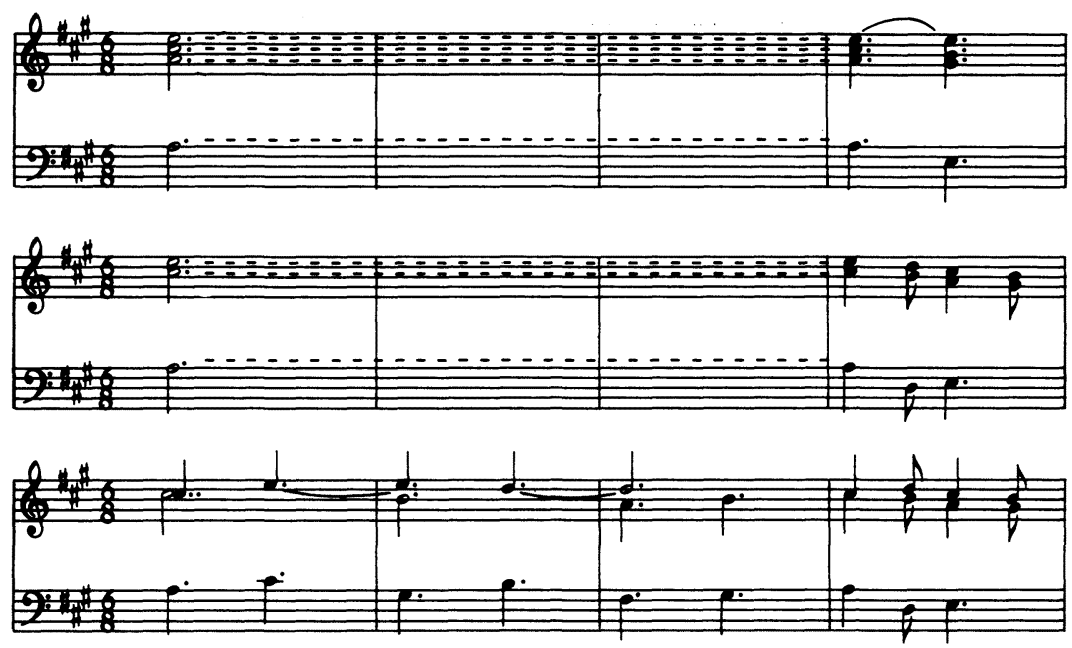

Exemple 4

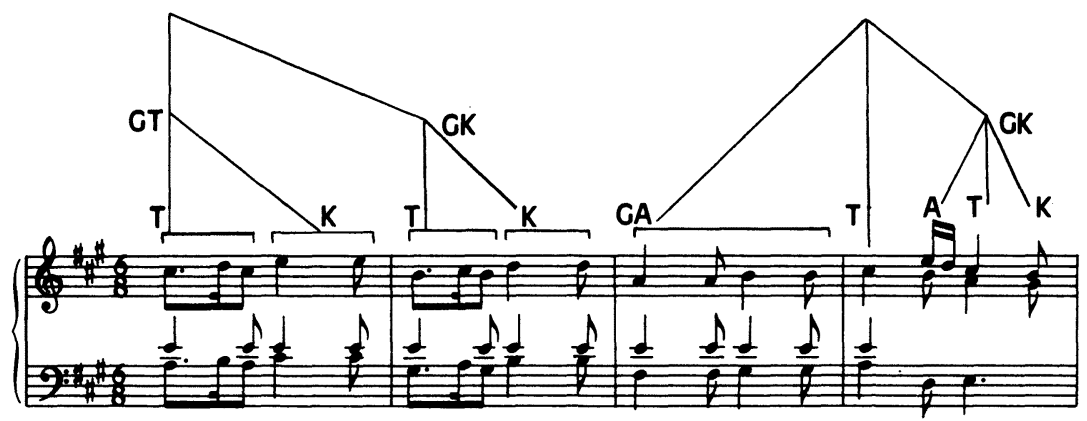

Exemple 5

2) BRAHMS. J'ai discuté dans mon ouvrage théorique déjà cité (chap. V) les douze premières mesures de cet Intermezzo dont on ne retrouve ici que le début statique. Je réduis, ici, l'exemple à trois mesures parce qu'elles englobent les deux interprétations. L'analyse schenkerienne du passage est moins instructive parce qu'on ne peut l'établir que sur un seul niveau (ex. 6): 


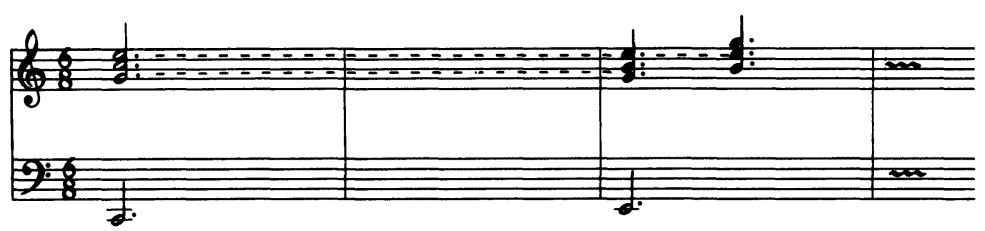

Exemple 6
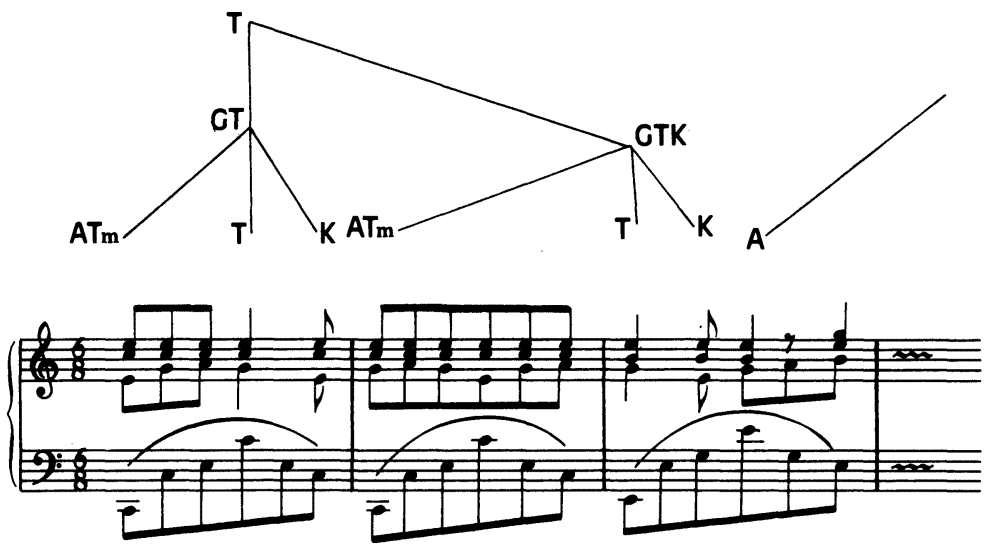

Exemple 7

Elle montre néanmoins la position de la désinence du groupe de longueur $15 / 8$ par la progression harmonique I-III et la descente de la ligne génératrice centrale do-si. Cette désinence est suivie d'un groupe arsique dont le repérage est facilité par le déplacement des trois lignes génératrices dans ce niveau, qui, de fait, est un Vordergrund. Remarquons en passant que le 15/8 devrait représenter une trame temporelle au sens où l'entendent Lerdahl et Jackendoff, et ce malgré l'absence de Vème degré fondamental (cf. infra). Le déploiement de la basse décrit un rythme formé de noires pointées que la prosodie ne peut ignorer: or les anacrouses des mesures 1 et 3 de l'exécution $b$ le font délibérément et tendent à produire un choc, une quasi-syncopation de la mélodie arpégée de la basse qui nie son propre rythme. Indépendamment de ces considérations touchant des rapports de hauteur, ce sont des considérations métriques et rythmiques qui m'amènent à l'application de la règle A.T.K. (ex. 7). Cette prosodie reconstitue le $6 / 8+9 / 8$ de l'interprétation a que d'ailleurs peuvent corroborer 
GPR 4 où le 9/8 est déjà une intensification du 6/8, et GPR 7 qui pourrait sans doute reconnaître une équivalence entre les rythmes des groupes T.K. du petit niveau, mesures 1 et 3 , eu égard à la stabilité. La théorie métrique de Lerdahl-Jackendơff ne reconnaîtrait toutefois par la distribution 6+9 du 15/8 comme je le propose: elle considèrerait vraisemblablement ces trois mesures comme régulières selon une alternance de mesures forte - faible - forte; mais l'analyse de l'exécution $b$ ne peut rejoindre ni mon analyse ni celle de la théorie en cours de description, puisqu'elle transforme le $15 / 8$ en $14 / 8$, qu'elle fragmente en un $5 / 8+4 / 8+5 / 8$ : soit une structure rythmique que ne peut assumer le composant métrique de base.

Il est temps d'en venir à présent à l'étude du composant métrique dans la théorie de Lerdahl-Jackendoff; nous l'avons évoquée au passage, et notamment par l'exemple 1 ci-dessus, qui est l'image hiérarchique que lui confèrent les règles de bonne formation. Les règles préférentielles, en dépit du caractère naturel dont les auteurs les déclarent pourvues, paraissent cependant être le fruit d'une intuition très acculturée.

Elles jettent parfois quelque doute sur leur spécificité métrique. La théorie s'articule bien sûr à partir du principe d'interaction entre mesure et groupe, et elle défend l'enracinement de celui-ci dans celle-là. Une appréhension plus phénoménaliste sera probablement amenée à déclarer l'inverse. Lerdahl et Jackendoff, en dépit de quelques restrictions qu'ils formulent eux-mêmes, confèrent à la métrique de grandes responsabilités.

MPR 5, par exemple, règle de longueur, est ainsi formulée dans sa version finale:

Préfèrer une structure métrique dans laquelle une battue relativement forte apparaît à l'insertion soit a) d'une hauteur relativement longue; b) d'une intensité de durée relativement longue; c) d'une liaison relativement longue; d) d'un pattern d'articulation relativement long; e) d'une durée de hauteur relativement longue aux niveaux adéquats de la réduction de la trame temporelle; f) d'une durée relativement longue d'une harmonie aux niveaux adéquats de la réduction de la trame temporelle (p. 84)..$^{19}$

Les propositions ne pourraient-elles être inversées, et ne concernent-t-elles pas davantage l'accent structural (thesis) que l'accent métrique? Pour ne reprendre que le seul point $f$ très important puisqu'il concerne le rythme de l'harmonie, aspect souvent négligé, il se pourrait que, sans dommage, on puisse 
l'énoncer sous la forme suivante: $H P R$ (où $H=$ Harmony): «Une harmonie de durée relativement longue aux niveaux de la réduction de la trame temporelle sélectionne de préférence un accent structural et éventuellement une battue forte de la structure métrique ".

Il se peut donc que le lecteur soit « sur ses gardes » en lisant le chapitre IV de l'ouvrage, la pire attitude serait cependant de passer outre sans en extraire la consistance. De fait, le poids métrique est démontré comme par l'absurde tout au long du chapitre: les barres de mesures sont éliminées des exemples. Il peut en résulter un joli désarroi: preuve de la nécessité de ce cadre synthétique tout au moins dans l'approche des oeuvres tonales monophoniques. Cette manière euphémique d'exprimer une réalité illustre des principes fondamentaux des règles qu'il convient d'isoler. Ainsi (p. 71), le choix du tactus comme niveau métrique le plus prégnant. A ce propos, il est noté que «le choix du tactus est lié au rythme de l'harmonie» (p. 74). Retenons la remarque: elle est primordiale en grammaire tonale et est susceptible de puissantes généralisations à toutes les structures, quoi qu'en pensent les détracteurs de Schenker qui voudraient infliger à leur adversaire une défaite sur ce point au nom de la sacro-sainte Mélodie. On isolera aussi la description de la syncope obtenue comme en creux par une règle qui déclare en substance (p. 76) qu'un événement sonore préfère une structure métrique à un niveau déterminé dont les battues fortes coïncident avec son insertion au même niveau. En faisant apparaître la syncope en négatif une telle formulation de la règle lui donne tout son poids. La syncope est aussi vieille que la polyrythmie, mais combien n'ont pas été perçues quand une métrique régulière n'en rappelait la puissance expressive. Ce n'est pas par hasard que le sens commun définit le jazz «musique syncopée »; beaucoup de musiques le sont bien davantage, mais ne comportent pas le beat qui définit la syncope comme violation de la règle pulsatrice.

Je ne voudrais pas quitter ce chapitre sans signaler une analyse particulièrement élégante du début du final du Quatuor, Op. 76, No 6, de Haydn: on y réfléchit profondément sur les interactions entre groupements et métrique. Une grammaire différente, fondée sur la priorité du groupe et de sa structure prosodique, inclurait forcément la structure métrique, mais sans en faire un principe d'enracinement. De mon point de vue, la vitalité de cet extrait provient en tout premier lieu de son organisation 
prosodique, laquelle révèle la métrique à l'auditeur, à partir de la mesure 5 seulement, la révèle donc régressivement. Je donne ci-dessous l'analyse prosodique que je propose du passage (ex. 8) sans autre commentaire. On remarque comment une telle lecture permet d'envisager de restituer la prosodie des structures polyphoniques. Cette superposition d'accent n'est pas ignorée dans la théorie de Lerdahl-Jackendoff qui la nomment " accents croisés ".20

Le lecteur aura compris, et cela deviendra encore plus clair dans un instant quand il sera question de la formalisation de la réduction des trames temporelles, que pour Lerdahl-Jackendoff, la structure métrique est la structure où s'enracine le groupe, en d'autres mots que ce composant est aussi important que le groupe, même s'il n'intervient que pour définir le niveau inférieur de l'édifice hiérarchique. De mon point de vue, le mètre ne serait qu'un rythme-cadre dans la structure prosodique du groupe, où, sans être nié, il n'aurait qu'une puissance relative. Tel est bien le sens de la divergence apparente. Toutefois, les faits ne sont pas aussi simples, et il ne peut s'agir, en la matière, de la défense d'un parti pris. Tout d'abord, il faut bien savoir que la définition prosodique du groupe, fondée sur la structure anacrouse-accent-désinence, est elle-même une définition soumise à une analyse de l'interaction des paramètres à l'intérieur des groupes et sous-groupes, et que parfois plus d'une définition est possible, d'où la diversité d'exécutions correctes d'un même passage. La règle débouche, en effet, directement sur l'interprétation de l'exécutant: c'est à partir de là que nous avons pu discuter les choix de phrasés dans les séquences de Mozart et de Brahms. En n'adoptant pas un tel point de vue, Lerdahl et Jackendoff entendent indiquer que leur intention est de fonder la hiérarchie des trames temporelles sur une structure régulière, correspondant à l'intuition de l'auditeur qui marquera le niveau inférieur du processus sonore qu'il entend par la battue régulière de la mesure. A cela, il n'y a auncune objection, le principe consiste à utiliser l'intuition en vue d'établir la hiérarchie sur une organisation bien formée, ce qui en soi est un progrès sur une lecture prosodique pouvant comporter des choix. Une difficulté se présente, toutefois, dès que l'on suppose que l'auditeur battant les temps de la mesure, les éprouve selon une alternance fort-faible. Je vois là bien plus la trace de théories naïves propres aux manuels de solfège, qu'une intuition réelle de l'auditeur qu'il soit ou non expérimenté. Ceci toutefois n'implique en rien que 


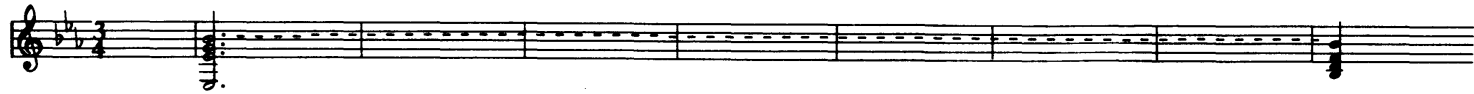
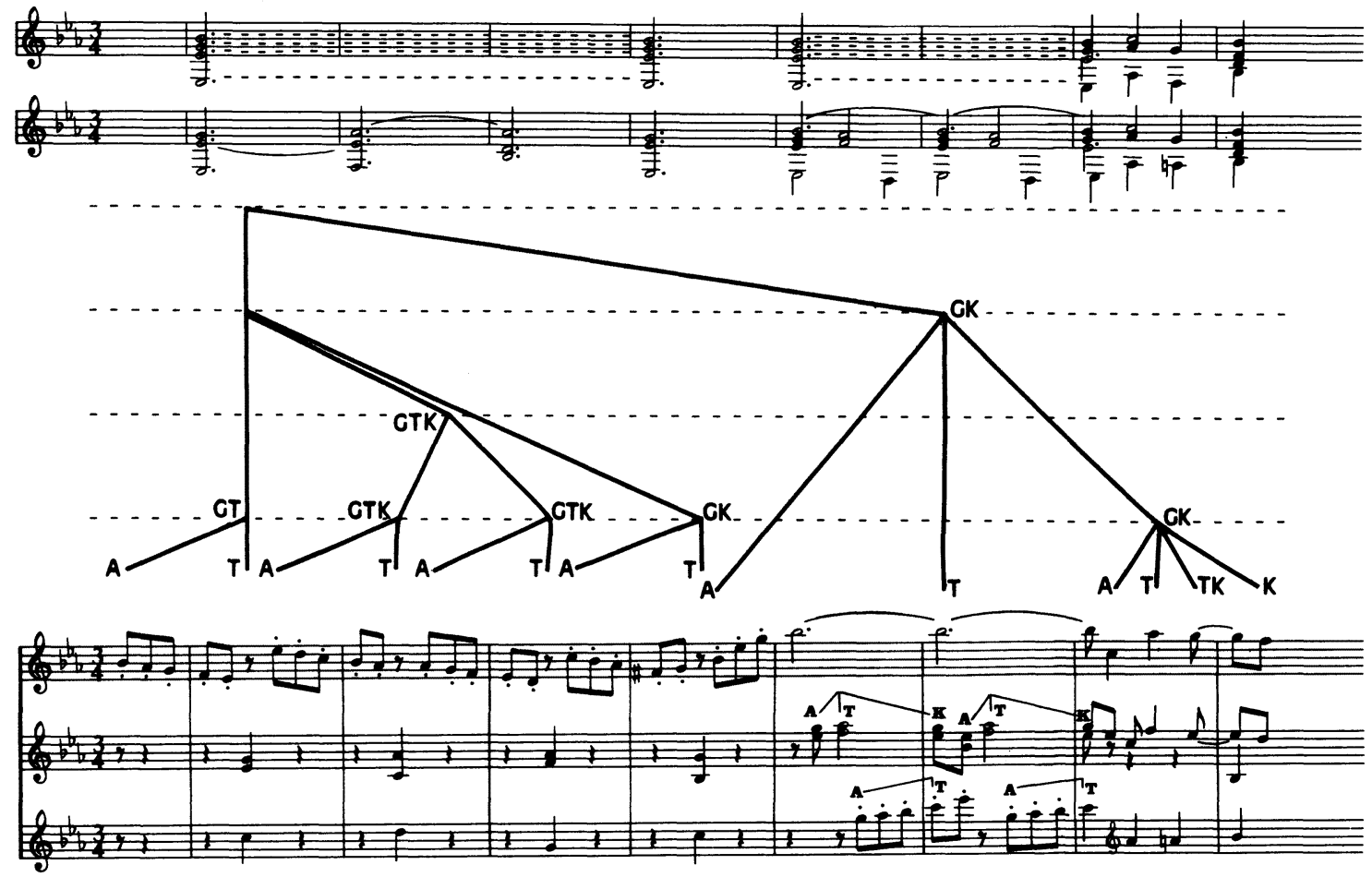

Example 8a 


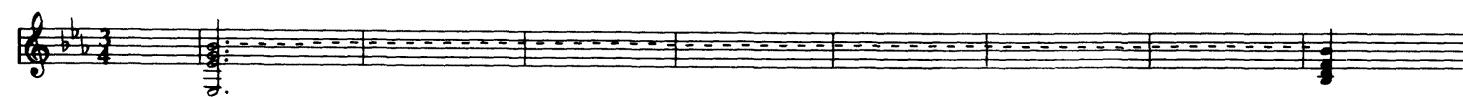

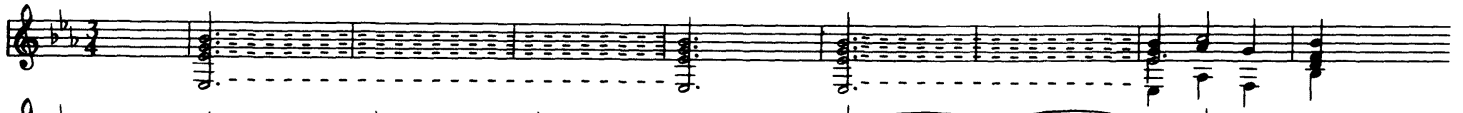
2

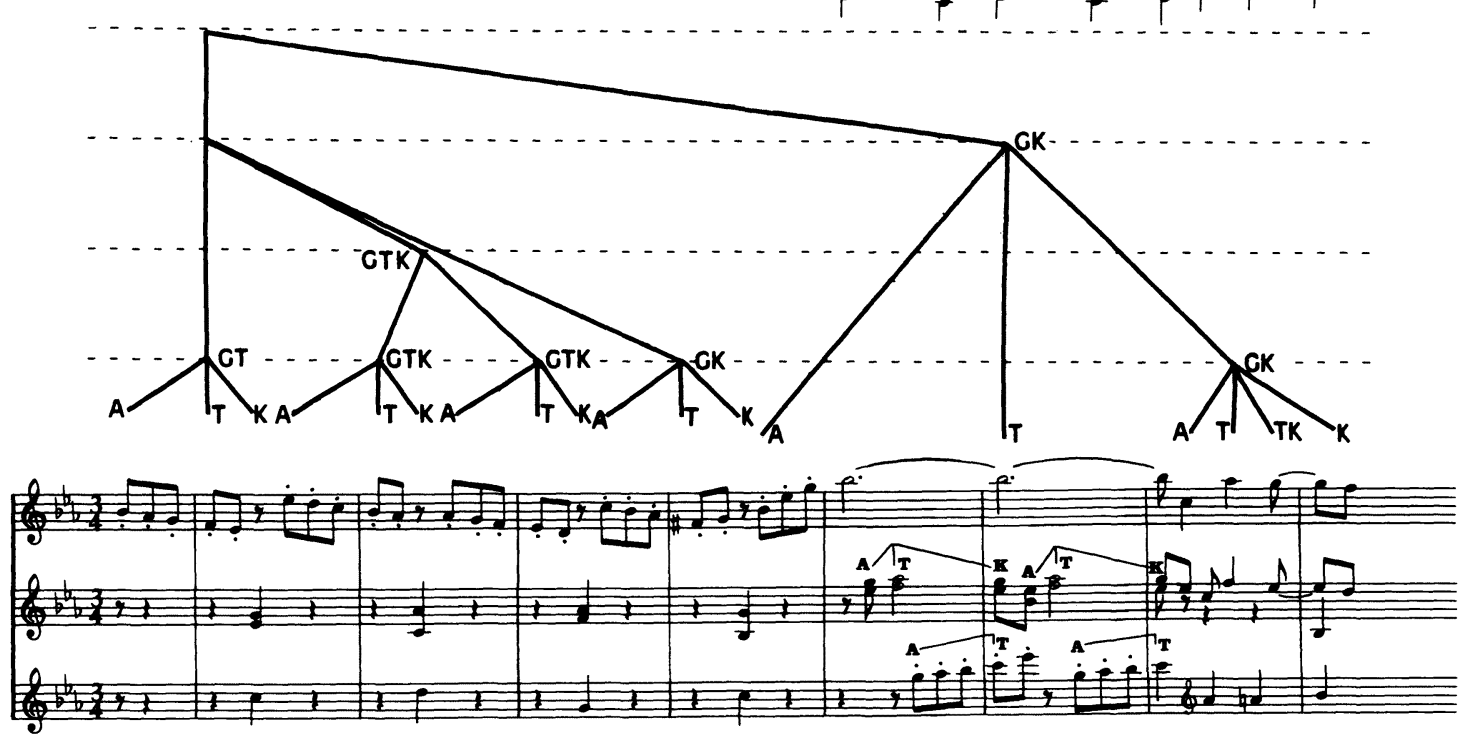


MWFR 3 et MWFR 4 (respectivement énoncées pp. 69 et 72) qui proclament chacune cette alternance au niveau de la mesure et du tactus, soient fausses. Pour leur donner une interprétation convaincante, je les déclarerai simplement exprimées dans un langage métaphorique. Car, en fait, ce que les auteurs font exactement - et il suffit pour s'en convaincre de se reporter à l'exemple 1-c'est exploiter les données de la notation proportionnelle dont le principe de base est la division binaire ou ternaire d'une valeur unitaire de durée: pour simplifier au maximum, appelonsla la ronde. Ayant admis une valeur étalon, ils déterminent le tactus dans la pièce soumise à l'analyse, lequel est le plus souvent égal à la valeur assignée par le dénominateur de l'indicateur de la mesure, puis ils sélectionnent un sous-tactus, lequel est déterminé par la plus petite valeur statistiquement significative utilisée dans le contexte. Cette interprétation étant admises, l'arbre qui peut être constitué à partir de l'exemple 1 peut être considéré comme l'image même de la hiérarchie rythmique de toute la polyphonie occidentale depuis le XIVème siècle et sa signification est probante indépendamment de tout recours à une quelconque intensité attribuée à la battue. Comme cela a été dit ci-dessus, l'arbre en question enregistre la progression géométrique, telle qu'elle est toujours présente, du sous-tactus à la ronde ou à la maxime. Il doit toutefois être clair que, en toute circonstance contextuelle, c'est le groupe qui reste le maître du jeu; d'où les irrégularités relevées par les auteurs eux-mêmes au niveau des hypermesures où le groupe détermine des réajustages (exemple: le début de la Symphonie en sol mineur de Mozart longuement discuté pp. 22 à 25). Il est hautement vraisemblable de supposer que les auteurs ont été induits vers une restauration de la notion de temps forts et faibles, très peu crédible aujourd'hui, par les théories prosodiques du langage exposées au chapitre XII (pp. 314 et sq.) où l'alternance des syllabes accentuées et non-accentuées et des pieds marqués et non-marqués est un phénomène bien réel dans la plupart des langues; l'extrapolation au phénomène musical n'en est pas moins risquée. Mais il me semble que l'interprétation des règles métriques, telle que je viens de la proposer, permet de restituer toute son efficacité à la théorie qui nous occupe, en cette matière particulièrement délicate. 


\section{Chapitres V à IX}

Les cinq chapitres centraux de l'ouvrage constituent le corps véritable de la théorie. ${ }^{21}$ Ils exposent les deux grands types de réduction dont il a été question plus haut: la réduction des trames temporelles et la réduction des prolongations. Je n'entreprendrai pas ici, bien sûr, une discussion de la controverse qui oppose un certain réalisme à l'empirisme logique au sujet de la réduction. Je crois que la description réaliste n'a pas dit son dernier mot, et qu'elle peut encore venir au secours de notre bonheur intellectuel; mais je crois davantage dans les moyens logiques de l'analyse que dans les descriptions foisonnantes, lorsqu'il s'agit de comprendre les mécanismes de génération de tout processus syntaxique quelle qu'en soit la nature. La réduction est apparue dans l'analyse syntaxique de la musique tonale avec la théorie schenkerienne il y a un demi-siècle; mais les musiciens sont restés prodigieusement indifférents à cet apport, et ils ont continué à produire des analyses, comme si rien ne s'était passé. Seuls, avant Lerdahl et Jackendoff, les disciples de Schenker ont maintenu le nouvel acquis. Lerdahl et Jackendoff reconnaissent explicitement leur det te à Schenker, mais ils placent au premier plan la structure rythmique qui, on le sait, ne fut pas l'objet d'une préoccupation dominante de la part du savant théoricien. D'autre part, ils évitent délibérément l'Urlinie (ligne génératrice) dans la réduction, ce qui est une dominante de la procédure dans la réduction schenkerienne. L'hypothèse de base défendue par les auteurs pour justifier la réduction est que "l'auditeur tend à organiser tous les événements sonores d'une pièce en une seule structure cohérente telle qu'ils sont entendus dans une hiérarchie d'importance relative» (p. 106). ${ }^{22}$ Deux conditions sont ajoutées à l'hypothèse sur la réduction:

a) les événements sonores sont entendus dans une stricte hiérarchie telle qu'elle se donne dans les groupements; b) des événements moins importants ne sont pas entendus simplement comme des insertions, mais dans une relation bien spécifiée à des événements environnants plus importants (ibid.) ${ }^{23}$

Comme dans la théorie schenkerienne, une importance particulière est attachée dans la réduction au point de départ et au but (cadence) du processus. Ces points forts sont justifiés psychologiquement (p. 107) par l'effet de masque qui, s'il survient, oblitère davantage la continuité à l'instant de la cadence qu'au centre. 
Puisque la réduction doit être celle de l'intuition de l'auditeur expérimenté, elle n'introduira pas d'autres éléments que ceux de la structure de surface: cette stratégie " est conséquente avec la méthodologie de la linguistique générative " où " habituellement les phrases sont prises telles quelles " (p. 112). ${ }^{24}$ C'est bien vrai, mais, cependant, on ne peut oublier le caractère souvent très abstrait de certains constructions de structures profondes en linguistique, auxquelles on peut être tenté de comparer des réductions musicales possibles construites à partir de divers transferts de voix dans la polyphonie. ${ }^{25}$

La réduction est formalisée au moyen d'une représentation en arbre; mais l'arbre choisi diffère totalement du modèle bien connu utilisé en linguistique. Il trace les différents niveaux de la réduction comme dans l'exemple 9 (début d'un choral de la Passion selon St Mathieu cité p. 115):
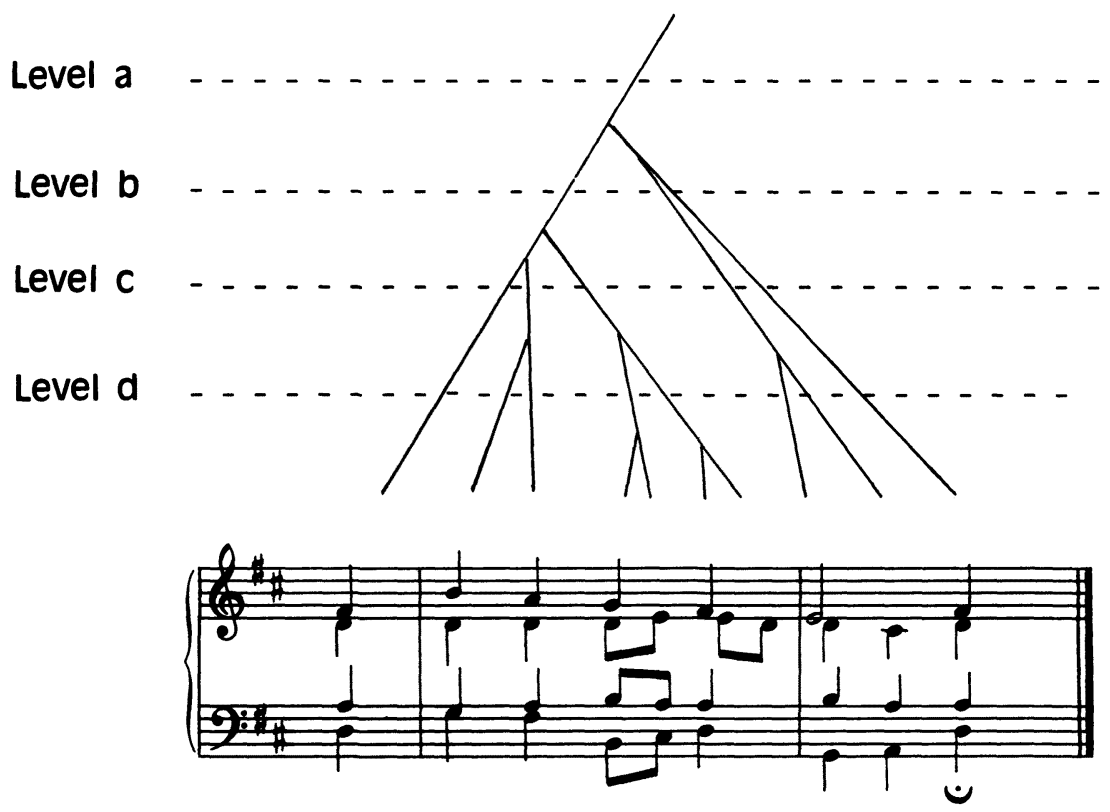

Exemple 9 
Dans ce type d'arbre une branche est tirée pour chaque entité sonore et les croisements sont prohibés. Aux branches les plus longues, représentant des événements principaux, sont attachées des branches plus petites exprimant la subordination d'événements moins importants à ceux qui les dominent. Une branche attachée à gauche exprime une élaboration d'un événement précédent. Depuis le traité d'harmonie de Schenker (1906), les rapports de subordination des degrés fondamentaux ont été explicités. ${ }^{26}$ Ils se traduisent par la position des fondamentales dans le cycle des quintes, par rapport à une tonique quelconque. Dans la présente théorie le même point de vue est conservé, mais les réductions gagnent en raffinement par le recours au critère de stabilité. Outre les relations entre fondamentales, les auteurs prennent en compte la dissonance considérée comme moins stable que la consonance, le retard ou l'appoggiature considérés comme moins stables que leur résolution, l'accord renversé moins stable que le même accord en position fondamentale, l'événement le plus stable étant forcément la tonique en position fondamentale (p. 117).

On peut naturellement tenter de falsifier une pareille proposition théorique, mais on verra que la cohérence interne de la théorie est telle qu'une telle tentative permet tout au plus de s'enfermer dans de nouvelles propositions, sans toutefois qu'une réfutation résulte de l'entreprise. Je vais ainsi poser une question que le lecteur considèrera comme fort légitime, et qui plus est l'est, en ce sens qu'y répondre favorablement ne peut manquer de produire des analyses correctes. Je m'interrogerai ainsi sur les niveaux de subordination. Plaçons en parallèle les deux systèmes de réduction de la théorie pour un même passage (ex. 10): a) réduction de la trame temporelle, b) réduction de la prolongation des mesures 1 à 4 de la Sonate de Mozart, K.331 (exemples cités pp. 120 et 122): 

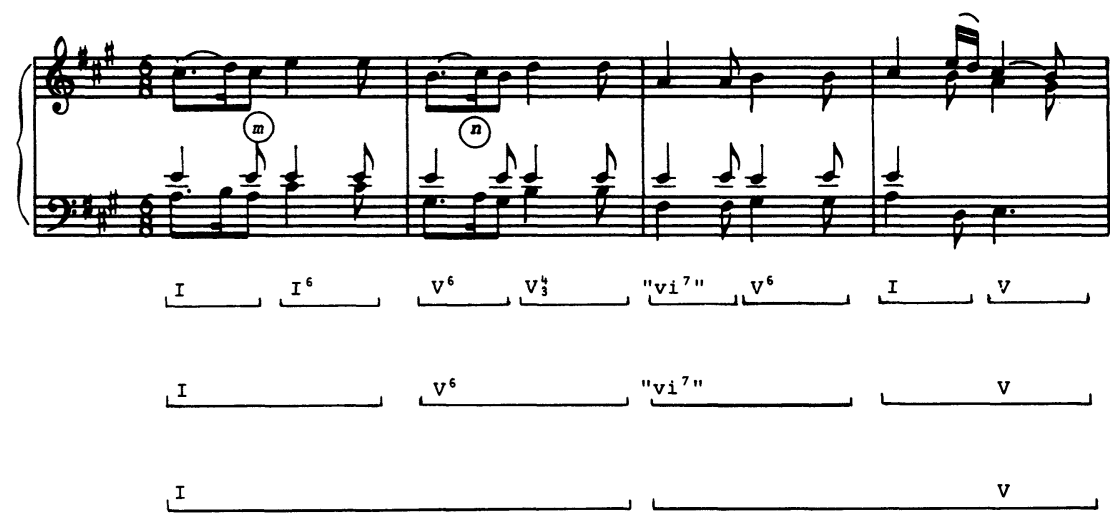

I

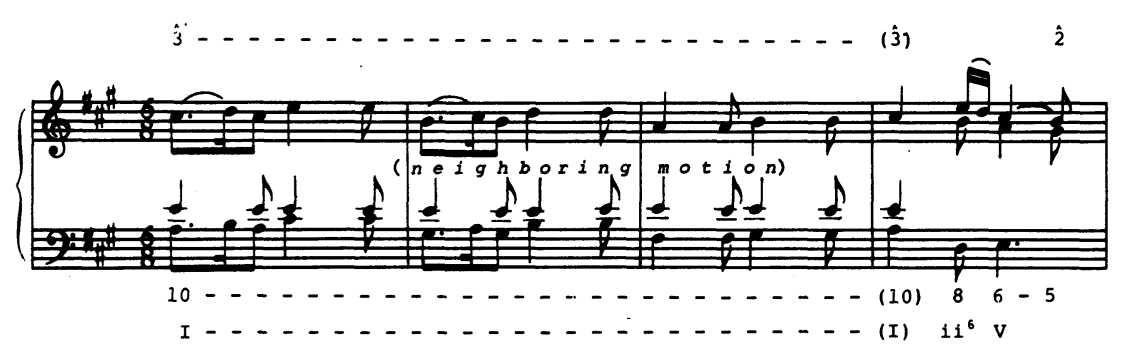

Exemple 10

Dans la première réduction, on lit quatre niveaux de groupements dominés par la structure métrique, les harmonies y sont inscrites sauf si leur durée est inférieure au niveau du tactus, la noire pointée. Dans la seconde, on lit sur un seul niveau une réduction de type schenkerien où les deux accords de tonique des premiers temps des mesures 1 et 4 sont reliés l'un à l'autre, après quoi apparaît la cadence. Les deux réductions se complètent mutuellement. La réduction de la trame temporelle "dit que des hauteurs particulières sont entendues en relation à une battue particulière dans un groupe particulier, mais elle ne dit rien sur la manière dont la musique s'écoule à travers ces segments" (p. 122) ${ }^{27}$; effectivement, ce dernier point est celui qui est pris en charge par la réduction de la prolongation. Si cette dernière réduction ne me pose aucun problème, la première par 
contre ne me laisse pas pleinement en repos. Je puis, en effet, y voir une prédominance de la structure métrique sur celle des groupes plutôt qu'une réelle interaction. Sans quitter le terrain de réflexion de Lerdahl et Jackendoff, je puis imaginer une exécution des mesures 3 et 4 qui organiserait préférentiellement les groupes de la manière précédemment décrite (cf. ex. 5). A nouveau, j'ai substitué la structure prosodique à la structure métrique. Mais celle-là, ne l'oublions pas, comprend celle-ci. Je défends cette solution parce qu'elle intègre dans la structure des groupes l'accord de Ilème degré de la mesure 4, membre initial de la demi-cadence. L'anacrouse de ce groupe désinentiel est légitimée par la battue faible du premier temps de la mesure 4, la suspension de la pédale de dominante, le point de départ d'une préférence du système harmonique sur le système contrapuntique pour marquer la cadence, et l'attaque sur une harmonie subordonnée de la dominante pouvant constituer une attaque de sous-groupe. Les auteurs, bien sûr, n'ignorent aucun des termes de cette argumentation, pas plus qu'ils ne peuvent ignorer la légitimité de mon désir. La remarque comporte la revendication de voir intégrer la totalité des membres d'une cadence harmonique dans la réduction de la trame temporelle. Cependant la logique même de leur grammaire doit les amener à rejeter mon amendement. Leur réponse tient en trois observations. La première a trait à la mission respective de la structure métrique et de celle des groupes:

Aux niveaux les plus petits, la structure métrique n'influence que le choix des trames temporelles et les trames sont de longueur régulière. A des niveaux intermédiaires, les limites des groupes peuvent interrompre la régularité imposée par le pattern métrique, et les trames temporelles résultent de l'interaction du mètre et du groupe. Aux niveaux supérieurs, les trames sont totalement déterminées par la structure des groupes, et la structure métrique est inopérante (p. 128). ${ }^{28}$

La seconde observation touche les cadences et les déclare réduites à deux membres dans le cas des cadences parfaites et rompues, à un seul, à fortiori, dans le cas de la demi-cadence (pp. 134 et 138). Enfin, la troisième, concerne les groupes entièrement contrôlés à partir des accents structuraux de débuts et de cadences (pp. 136 et 137). Puisque ce sont les événements les plus stables qui sont porteurs d'un accent structural, la structure arborescente des groupes ne traduit qu'un jeu de relations entre toniques et dominantes, à la manière de l'Ursatz schenkerien 
(p. 139). Le lecteur aura certainement compris que le Ilème degré subordonné de la dominante dans les cadences ne peut être enregistré qu'au niveau métrique dans la réduction des trames temporelles.

Cet événement sonore ne trouvera la place que je rêve de lui voir attribuer que dans la réduction de la prolongation. Toute difficulté n'a pas pour autant disparu: dans la formalisation des mesures 7 et 8 du même mouvement de sonate (p. 156).
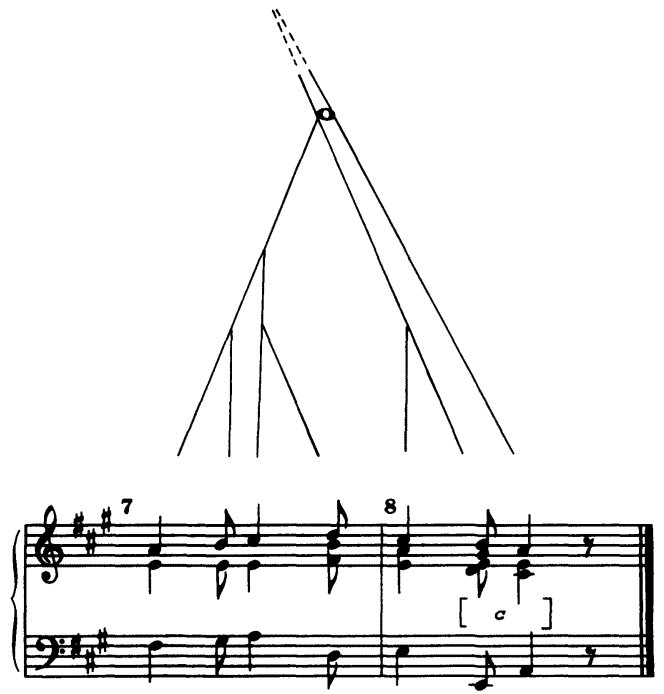

Exemple 11

Le IIème degré de la dernière battue, mesure 7, est montré comme subordonné de la tonique qui le précède probablement pour la raison sus-mentionnée suivant laquelle seule la structure métrique précise la position des événements au niveau inférieur. Quoi qu'il en soit, même à supposer que l'on ne reconnaisse pas ce degré comme appartenant à la cadence, on ne peut lui refuser, en fonction de sa position dans le cycle des quintes, sa subordination au Vème degré qui le suit plutôt qu'à la tonique qui le précède; la théorie qui nous occupe a bien spécifié, dès le début, que la structure métrique et la structure des groupes pouvaient être hors phase. Une règle de segmentation de la trame est d'ailleurs contenue dans la grammaire et prévoit dans les cas d'absence de phases la trame augmentée: elle spécifie que 
... dans une structure de groupement sous-jacente, si une limite de groupe intervient entre une battue et la battue précédente du même niveau, la battue détermine une trame temporelle augmentée qui résulte de l'intervalle compté à partir du groupe jusqu'à la fin de la trame régulière (règle 2c, p. 147). ${ }^{29}$

Notre divergence de vue pourrait tourner autour de ces mots "battues de même niveau »: noire-croche, la condition n'est pas remplie. C'est vrai, mais le niveau le plus prégnant, celui du tactus, inclut les deux valeurs. Je crois que la discussion porte ici davantage sur le sens donné à la conception de la cadence. Comme Schenker, tout au moins au niveau de l'Ursatz, les auteurs ne reconnaissent pas la puissance conclusive du membre antépénultième, non, certes, inéluctablement présent en tout contexte, mais d'une incidence inévitable dans les nombreux cas où il apparaît. Au nom de ce qu'il nommait "le triangle sacré ", Schenker le refoulait dans son Mittelgrund, mais il me semble que, moyennant peu d'aménagements, la cadence pourrait dans la présente théorie, où Dieu merci le mysticisme n'a pas cours, inclure le (ou les) terme(s) subordonné(s) de la dominante.

Il est impossible de décrire ici les détails de procédure de formalisation de la réduction des trames temporelles: relevons simplement que cette procédure suit comme précédemment une série de règles de bonne formation sur lesquelles se greffent les règles préférentielles de perception. L'arbre se construit nécessairement du bas vers le haut, étape par étape. Les niveaux les plus petits s'élaborent en fonction de la progression géométrique donnée par la grille métrique. Quand le tracé atteint le niveau des groupes, c'est l'accent structural qui assigne une tête (b-beginning) et une fin (c-cadence) à la trame ou une union de trames temporelles (time-span(s)). Les têtes de trames peuvent donc aussi bien être des accents de début que des termes ultimes de cadences. Au sommet, les jeux de subordination ont lieu entre accents, soit que le début est subordonné à la cadence, ou, plus rarement, que se produise le contraire, et ceci à tous les niveaux, pouvant s'étendre jusqu'à la pièce entière (cf. principalement pp. 158 et 159). L'incidence des règles préférentielles est forcément des plus variable selon les contextes, selon aussi leur origine: mètre, harmonie, parallélisme, stabilité, etc. (cf. principalement pp. 159 à 174).

'Parvenu à ce point de l'exposé, ce qui importe le plus devient le test. En dehors de ceux qui concernent le psychologue, j'en suggèrerai deux: l'un restant rigoureusement à l'intérieur de la 
théorie, est assez proche de ce que les auteurs proposent dans le chapitre $\mathrm{X}$ de l'ouvrage: il consiste à soumettre des textes riches à l'ensemble bien répertorié de toutes les règles lues comme algorithmes, et à sonder les conflits que la complexité des contextes devrait mutliplier. L'autre devrait permettre une comparaison avec l'analyse schenkerienne sur base de contextes très classiques. Ce second test révèlerait certainement une élégance de formalisation de la part de Lerdahl et Jackendoff que la logique de Schenker a été totalement impuissante à concevoir; mais aussi, probablement, il mettrait en évidence qu'un Vordergrund schenkerien bien construit engendre des patterns éventuellement moins proches du perçu, mais plus proches de la poétique musicale classique. Si le compositeur est le premier auditeur expérimenté de son oeuvre, avant de l'entendre il la conçoit selon des normes syntaxiques et stylistiques en usage à son époque, ou qu'il a mis en oeuvre lui-même; d'où si le test suggéré est positif, de mon point de vue, la nécessité d'accroître le nombre de règles de bonne formation et de concevoir des règles préférentielles d'élaboration en complément de celles que nous devons à Jackendoff et Lerdahl.

Il est temps d'en venir à présent à la réduction de la prolongation. Les familiers de la théorie schenkerienne n'auront pas de peine à l'assimiler, dans la mesure où cette partie de la théorie se greffe tour à tour sur l'Hintergrund ou le Mittelgrund schenkerien, tout en pouvant incorporer les détails du Vordergrund. Ce qui, par contre, ne se retrouvera pas, on l'a noté, c'est l'Urlinie qui manifestement n'emporte pas l'adhésion des auteurs. D'emblée, des raffinements complémentaires sont introduits: puisque la théorie de Lerdahl et Jackendoff est d'abord une théorie de psychologie cognitive, les rapports de subordination des fondamentales harmoniques à travers le cycle des quintes seront exprimés en termes de tension et de relaxation (pp. 179-83). Ainsi, ce que j'appelle pattern convergent inverse (VII-III-VI-IIV-I) exprimera la relaxation. Mélodiquement de même, une ligne ascendante donc, s'écartant de la tonique, traduit une tension, alors qu'une ligne descendante y ramenant, traduit une détente. En outre, trois types de prolongation sont pris en compte: 1) la forte prolongation, laquelle est manifestée par deux événements identiques de l'harmonie quelle que soit leur distance dans une région donnée ; 2) la faible prolongation exprimée par deux harmonies disposées différemment, mais ayant une fondamentale identique ; 3) la progression, représentée par deux événements 
harmoniques différant par la fondamentale, toujours quelle que soit leur distance dans la région observée, celle-ci pouvant atteindre la dimension de la pièce. L'arbre de réduction de la prolongation traduit ces trois catégories dans la notation: un cercle vide symbolise la première, le même cercle rempli indique la seconde, la troisième ne comporte aucun symbole (pp. 183188). Contrairement à l'arbre de réduction des trames temporelles, celui-ci se construit du haut vers le bas, ce qui se conçoit aisément si l'on songe que les trois catégories de prolongation sont inventoriées successivement en partant du repérage des événements les plus importants (p. 188).

Le problème le plus central et le plus délicat qui se pose tout au long des deux chapitres consacrés à la réduction de la prolongation est celui des relations de cette réduction avec celle des trames temporelles; et au terme de la lecture, le sentiment éprouvé est que si cette recherche théorique est poursuivie, la tâche la plus urgente reste une clarification complémentaire du problème. La relation entre les deux réductions est immédiatement évidente puisque dans l'une comme dans l'autre, des rapports de subordination se traduisent dans l'arbre par des branchements à gauche ou à droite d'événements dominants qui expriment des rapports de hauteurs principalement harmoniques. De fait, la réduction de la trame temporelle sert d'input à la réduction de la prolongation.

La forte dépendance de la réduction de la prolongation, par rapport à la réduction de la trame temporelle, constitue une prédiction majeure de notre théorie. Elle affirme que les patterns perçus de tension et de relaxation dans les structures de hauteurs dépendent crucialement de la hiérarchie des événements structuralement importants dans les trames temporelles tels qu'ils sont définis par le mètre et le groupement. En d'autre mots, la compréhension par l'auditeur de l'enchaînement des hauteurs dans une pièce, est fonction de la manière dont il en segmente la surface (p. 188). ${ }^{30}$

Voici deux arbres traduisant l'Ursatz schenkerien dans les deux réductions: 
a

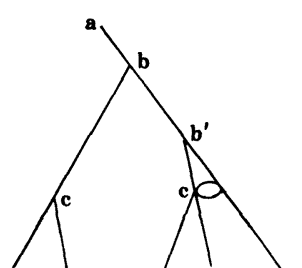

b

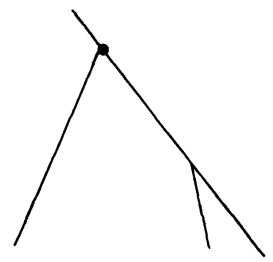

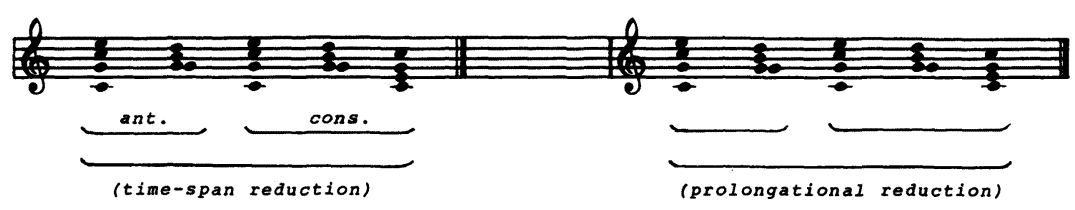

Exemple 12

Ils sont ainsi commentés:

Bien que ces arbres se regardent de façon similaire, ils reçoivent une interprétation différente: a) exprime la courbe du mouvement tonal du commencement structural à la cadence de la pièce; b) dit tout d'abord que la pièce forme une prolongation de tonique allant dans le sens de la détente, et ensuite que la tonique initiale se tend vers la dominante qui se relache plus fortement que la tension qui précède vers la tonique finale (p. 189). ${ }^{31}$

Cet exemple révèle un degré minimal de non-congruence entre les deux réductions, mais très souvent ce degré se renforce du fait que dans la réduction de la prolongation, la progression tonale est évaluée en échelle de divergence et de convergence par rapport à une tonique (ou si l'on préfère en degrés de tension et de détente), il en résulte dans la réduction de la prolongation un type d'arbre où les branchements droits et gauches s'équilibrent, exprimant ce que les auteurs dénomment une forme normative (pp. 197-201), forme que l'arbre de la réduction des trames temporelles n'a pas mission de refléter. Cependant, on l'a noté, la théorie reconnaît la dépendance des deux réductions: la première est l'input de la seconde, et une hypothèse de base est ainsi énoncée:

Le choix des événements qui définissent les régions de prolongation, est fortement influencé par l'importance relative des événements dans la réduction de la trame temporelle (p. 213). ${ }^{32}$

De plus, la première règle préférentielle de prolongation, spécifie: PRPR.1 ... dans le choix de l'événement le plus important de 
la prolongation $e_{k}$ d'une région de prolongation $\left(e_{i}-e_{j}\right)$ préfère fortement un choix dans lequel $e_{k}$ est relativement le plus important dans la trame temporelle (p. 220). ${ }^{33}$

Mais, ici comme ailleurs, les règles préférentielles entrent en conflit avec d'autres, et PRPR 1 qui vient d'être citée est généralement englobée dans un Principe d'interaction avec PRPR 3 qui, pour l'événement $e_{k}$, choisit l'enchaînement le plus stable avec un des points d'aboutissement dans la région prolongationnelle $\left(\mathrm{e}_{\mathrm{i}}-\mathrm{e}_{\mathrm{j}}\right)$ (p. 224).

Jusqu'où peuvent aller les divergences entre les réductions du fait de l'action du principe d'interaction entre les règles (pp. 227-232)? Revenons avec les auteurs à l'exemple mozartien privilégié (K.331, mes. 1 à 4). L'exemple 13 (cité p. 227) montre la réduction de la trame temporelle; l'exemple 14 (cité p. 231) propose deux possibilités de réduction de la prolongation, a et $b$.

Quelles remarques peut inspirer la comparaison de ces schémas?

1) Ma première constatation est que la réduction de la prolongation (ex.14) est la plus pertinente pour l'analyse syntaxique du fragment. Bien que, dans le principe, sa mission ne la conduise pas à sélectionner des groupes, elle en sélectionne avec une précision maximum sur quatre niveaux donnant les répartitions $1+2+3+4$.

2) L'exemple 14a, pertinent à l'intérieur de la théorie décrite, ne l'est pas pour l'analyse. La branche de l'harmonie de VIème degré, mesure 3, rattachée à la dominante qui la précède et non, comme en b, à celle qui la suit. Or il s'agit d'une harmonie dissonante $\mathrm{VI}^{7}$ qui, de ce fait, tend vers sa résolution et, donc, peut difficilement justifier sa subordination à un événement qui la précède immédiatement.

3) En comparant les exemples 13 et 14, on note que, dans la réduction de la prolongation, les niveaux ne sont pas étiquetés, ceci conformément à l'intention exprimée par les auteurs. Cette structure n'est pas assez régulière: «ce qui compte ici, ce sont les patterns d'enchaînement et de subordination.... La signification d'un événement est fonction de son degré d'enchâssement dans la structure dominante» (p. 203). ${ }^{34}$

4) Seuls les niveaux $a$ et $b$ de l'exemple 13 et les branchements supérieurs de l'exemple 14 sont congruents; ils esquissent le tracé d'un Ursatz et d'un Mittelgrund schenkerien; le Mittel- 

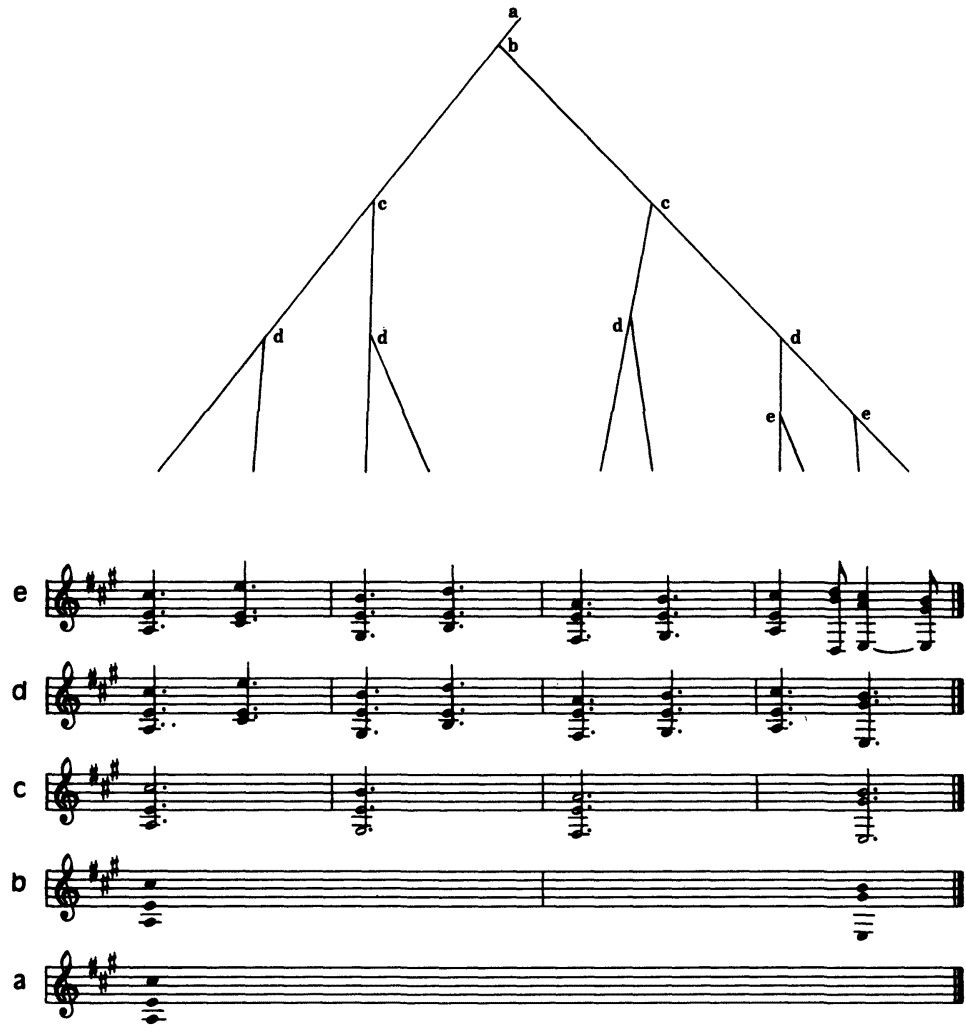

Exemple 13

a

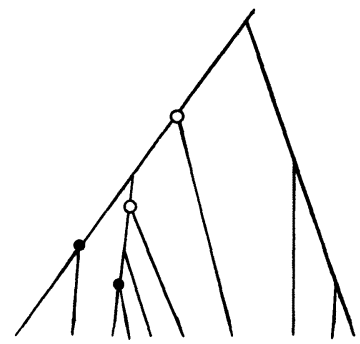

b

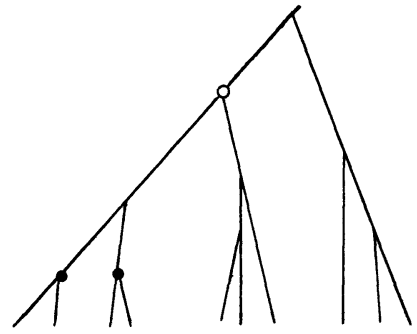

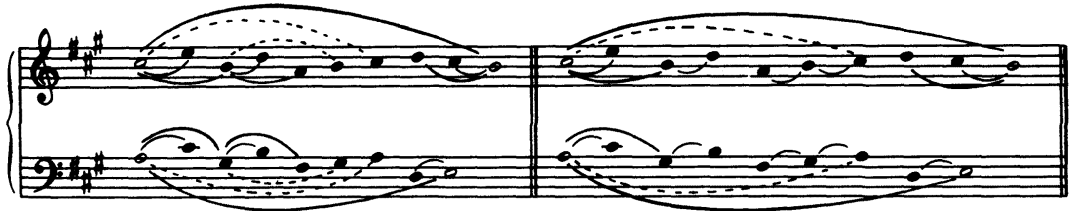

Exemple 14 
grund étant toutefois plus complet dans l'exemple $14 \mathrm{du}$ fait de l'enregistrement du IIème degré de la cadence, point discuté ci-dessus.

5) Au niveau C, la réduction de la trame temporelle enregistre, conformément à la théorie, le groupe de début et le groupe de fin. Aux niveaux inférieurs, elle ne peut incorporer de plus petits groupes, elle trace la grille métrique en reliant les événements aux deux groupes centraux.

Deux conclusions paraissent pouvoir se dégager des implications de ces constatations: a) des groupes de petits niveaux pourraient n'apparaître que par chance dans les petits niveaux de l'analyse dans la réduction des trames temporelles; ils risquent de ne pas apparaître davantage dans les petits niveaux de la structure des prolongations, dans la mesure où celle-ci unifie des aires harmoniques semblables, transcendant les groupes. Le malentendu - si malentendu il y a - ne naît-il pas au moment de l'énoncé de la motivation de la réduction de la prolongation (p. 121) à l'instant où sont citées les quatre premières mesures de la Sonate, Op. 2, No 3, de Beethoven, et où est rappelé le début de la Symphonie en sol mineur de Mozart, deux exemples où une même harmonie ( $V$ chez Beethoven, II chez Mozart,) franchit incontestablement les limites des groupes? Là sont dévolues les missions propres à chacune des réductions: l'une, celle des trames temporelles contrôlera les limites de groupes; l'autre, celle des prolongations mettra en évidence la continuité harmonique et mélodique. Mais précisément la cohérence de la théorie n'est-elle pas là? Certes ... Mais il doit être permis de souhaiter de plus forts liens de connivence entre les deux réductions. Est-ce que le prolongement d'une harmonie au-delà des limites de groupes a le même sens à tous les niveaux? Est-ce que de petits groupes peuvent être exclusivement contrôlés par une grille métrique en étant simplement rapportés à une relation de tonique à tonique, de tonique à dominante ou de dominante à tonique au niveau supérieur? Nous avons appris que la réduction de la prolongation est au moins partiellement dérivée de celle de la trame temporelle; faut-il en rester là? Les input ne peuvent-ils s'inverser et la structure prolongationnelle ne peutelle inspirer le repérage de l'organisation des groupements? Cette inspiration pourrait, par exemple, moyennant certaines coopérations entre règles préférentielles permettre de réorienter certaines branches dans l'arbre des trames temporelles. J'ai si- 
gnalé le problème du membre précédent la dominante dans les cadences, mais je suis non moins perplexe face à la subordination de la tonique (mes. 21) à la dominante initiale dans l'analyse du premier mouvement de l'Op. 31, No 2 de Beethoven (p. 256) sauf, bien entendu, si un rattachement postérieur est postulé, mais les auteurs présentent très justement les vingt premières mesures de cette sonate comme une large anacrouse structurale: dès lors, pourquoi ne pas avoir inversé les branchements? On se posera peut-être aussi des questions sur l'absence d'étiquetage des niveaux dans la réduction des prolongations: étiquetés ou non, les niveaux sont présents. L'une des déficiences de l'analyse schenkerienne est précisément de ne les avoir notés qu'implicitement en dépit d'une graphisme qui tendait à les expliciter. Avec le même graphisme, Allan Forte (1982) parvient à une explication hiérarchique infiniment plus claire; il serait de ce point de vue intéressant de soumettre certaines de ses analyses de pièces complètes à une réduction des prolongations figurée dans un arbre. Du même point de vue, le lecteur de l'ouvrage de Lerdahl-Jackendoff notera, par exemple, le support efficace que constitue le rappel des niveaux $a, b$ et $c$ de la réduction des trames temporelles dans la réduction de la prolongation de la mélodie de Schubert Morgengruss (p. 267). Quand une zone de forte prolongation est enregistrée à un niveau relativement élevé, le mode de subdivision de la zone en question dans les niveaux inférieurs, dénote un découpage de groupes que la réduction des prolongations peut mettre en évidence avec une particulière efficacité.

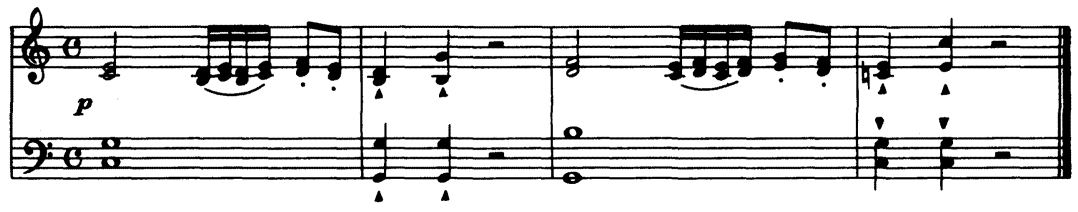

Exemple 15

Ainsi, dans le début de l'Op. 2, No 3 de Beethoven dont il était à l'instant question (cf. ex. 15), il est plus informatif de savoir que la zone harmonique de dominante qui domine les mesures 2 et 3 se divise en deux parties d'égale longueur, dont la première (mesure 2) est subordonnée à la tonique de la mesure 1, et la seconde (mesure 3) subordonnée à la tonique de la mesure 4: cette subdivision et ce mode de subordination venant de la con- 
figuration mélodique et du passage de l'état de consonance (mesure 2) à celui de dissonance (mesure 3) importent plus que de savoir qu'une zone de dominante franchit les groupes de deux mesures. Une observation analogue peut être faite à propos de Morgengruss où la réduction de la prolongation (p. 267) transmet aussi clairement que la réduction des trames temporelles (p. 265), le groupement contenu dans les mesures 8-10 enchâssé dans la zone de dominante englobant les mesures 5-11.

Ces diverses remarques n'ont manifestement pas échappé aux auteurs; je leur donne simplement une interprétation qu'elles n'ont pas reçue dans leur théorie. Elles tendent, de ma part, à rapprocher la portée des deux réductions en accentuant peut-être le poids de la seconde (prolongations). Ma conviction profonde est que les deux réductions peuvent parler d'une seule voix quand il s'agit de décrire la hiérarchie des groupes. Si on refuse ce complément de poids à attribuer à la réduction des prolongations, on risque de lui faire dire parfois trop peu, mais aussi parfois trop. Ainsi quand ils définissent les formes par la réduction des prolongations, les auteurs acceptent quelques interprétations qui semblent nier partiellement ces formes mêmes. Définissant la forme binaire baroque (pp. 241 et 242), ils considèrent comme correct que la dominante par laquelle débute la deuxième section au-delà des barres de reprise soit une élaboration (au sens anglais du terme) de la dominante d'aboutissement. Or, en se plaçant au plan psychologique qui fonde cette réduction - la relation tension/détente - il est difficile, pour des questions de longueur tout d'abord, de ne pas éprouver, comme amorce de détente, le début des secondes sections des pièces baroques, à l'exception de petits divertissements momentanés, tels qu'il s'en trouve dans les Sonates de Scarlatti. Comme la réduction des trames temporelles, la réduction des prolongations devrait préférer le rattachement de la dominante ou de tout autre degré immédiatement consécutif aux barres de reprises à une tonique de la deuxième section.

Il peut sembler paradoxal d'en appeler à une convergence de représentation des deux réductions, alors que tout au long de l'ouvrage, l'argumentation s'attache à démontrer que la richesse et la complexité d'un processus musical résulte 1) de l'absence de relation de phase entre composant métrique et composant de groupes ; 2) de l'accroissement du nombre de conflits entre règles préférentielles; 3 ) de la non-congruence entre les deux réductions. Quand (p. 289) les auteurs en arrivent à définir ce que 
pourrait être un archétype musical à partir de leur théorie, une telle structure n'admet aucun conflit ni aucune absence de concordance de phase; et l'on devine derrière un tel archétype, la musique la plus banale et la plus triviale que l'on puisse imaginer. Que peut, dès lors, signifier ce souci d'une relation plus étroite entre les deux réductions? Ne revient-il pas à vider toute la théorie de son contenu? Sans doute ne peut-il s'agir de promouvoir un type d'analyse qui tendrait à modifier les structures que la musique exhibe, et à en camoufler la richesse et la complexité. Simplement et normalement, je me situe dans la perspective d'une étape ultérieure de la recherche, étape qui permettrait une lecture explicite de faits qui dans le cadre de constatations séparées demeurent aujourd'hui implicites. On note avec précision des divergences structurales entre les réductions, on enregistre des conflits de règles, mais il reste extrêmement difficile, dans le cas d'oeuvres importantes, de dépasser ce degré d'une identification des situations par la concrétisation d'une synthèse. Cette étape pourrait peut-être être celle du devenir le plus immédiat dans ce cadre de recherche, mais il y faudra autant de prudence que de savoir-faire.

Ici s'achève ce tour d'horizon. A Generative Theory of Tonal Music est bien l'un des ouvrages les plus forts écrits sur la musique que j'aie lu depuis très longtemps. Si j'ai été amené à en discuter certains détails, c'est en raison des hypothèses audacieuses qu'il contient. La discussion critique, elle aussi, du reste - et surtout quand il s'agit d'une recherche aussi neuve et d'une telle ampleur - comporte ses risques: les assumer est le plus bel hommage que l'on puisse rendre aux oeuvres appelées à laisser leur trace. Les points controversés me paraissent de peu de poids en regard de l'acquis global de l'ouvrage indubitablement irréversible, et qui devrait constituer le fond de multiples greffes. De ce point de vue, et au risque de surprendre, je souhaiterais que le premier lecteur concerné soit le compositeur. Les auteurs eux-mêmes y ont d'ailleurs songé: les quelques remarques, nullement réactionnaires, qu'ils font sur la musique contemporaine (pp. 296 à 301), ne visent en rien à le ramener au système tonal dont ils savent pertinement qu'il a vécu; elles sont plutôt une invitation à réfléchir sur la nature de la perception humaine, et discrètement aussi, elles rappellent l'importance de certaines hiérarchies structurales. Au point culminant de la réflexion, on lit: 
La plupart des théories sur la musique contemporaines se sont préoccupées soit de la description du système compositionnel, soit de la systématisation des procédures analytiques, sans les relier directement à la compréhension de l'auditeur. Notre démarche insiste sur la distinction cruciale qu'il y a entre les principes au moyen desquels une pièce est composée et les principes au moyen desquels elle est entendue (c'est-à-dire ceux qui permettent à l'auditeur de construire une représentation mentale de la pièce). Comme théoriciens, nous ne sommes concernés que par ces derniers (p. 300). ${ }^{35}$

La question est bien sûr de savoir si une grammaire générative peut être plus qu'un algorithme, et si l'on peut en attendre un salut pour la composition. J'ai semblé y croire (Deliège 1974) mais Nattiez aujourd'hui encore se méfie d'une telle proposition (1983). Il y eut pourtant Webern, émetteur d'une grammaire générative, implicite seulement, il est vrai. Aujourd'hui peutêtre l'ouvrage ici présenté peut-il révéler de nouvelles ressources? Quel compositeur découvrira le moyen d'articuler des hiérarchies nouvelles engendrant quelqu'idiome inédit par une réflexion approfondie sur le système qui le plus s'identifie à nos traditions musicales? Rêve... utopie... délire! Mais les fondations les plus solides et les plus durables sont bien aussi, je crois, de type syncrétique! 


\section{NOTES}

1. Les travaux du colloque n'ont pas été publiés.

2. David Epstein, musicologue dont les travaux se situent au carrefour des théories schenkerienne et schoenbergienne, est l'auteur d'un ouvrage important d'analyse musicale, Beyond Orpheus (1979).

3. "At the present stage of development of the theory, we are treating all music as essentially homophonic; that is, we assume that a single grouping analysis suffices for all voices of a piece. For the more contrapuntal varieties of tonal music, where this condition does not obtain, our theory is inadequate. We consider an extension of the theory to account for polyphonic music to be of great importance. However, we will not attempt to treat such music here except by approximation".

4. "A piece of music is a mentally constructed entity, of which scores and performances are partial representations by which the piece is transmitted.... In our view, the central task of music theory should be to explicate this mentally produced organization. Seen in this way, music theory takes a place among traditional areas of cognitive psychology such as theories of vision and language".

5. "... his conscious grasp of musical structure; an acculturated listener need never have studied music. Rather we are referring to the largely unconscious knowledge (the "musical intuition") that the listener brings to his hearing - a knowledge that enables him to organize and make coherent the surface patterns of pitch, attack, duration, intensity, timbre, and so forth. Such a listener is able to identify a previously unknown piece as an example of the idiom, to recognize elements of a piece as typical or anomalous, to identify a performer's error as possibly producing an "ungrammatical» configuration, to recognize various kinds of structural repetitions and variations, and, generally, to comprehend a piece within the idiom".

6. "The two idealizations we have adopted, that of the experienced listener and that of the final state of his understanding, are comparable to idealizations made elsewhere in cognitive psychology. Without some initial simplification, the phenomena addressed by scientific inquiry have almost always proved intractable to rational investigation".

7. «... it would be fruitless to theorize about mental processing before understanding the organization to which the processing leads. This is only a methodological choice on our part».

8. "A formal theory of musical idioms will make possible substantive hypotheses about those aspects of musical understanding that are innate; the innate aspects will reveal themselves as « universal » principles of musical grammar».

9. «Formalism alone is to us uninteresting except insofar as it serves to express musically or psychologically interesting generalizations and to make empirical issues more precise». 
10. Le terme "prolongation" est issu directement de la théorie de Schenker qui d'ailleurs fournit le modèle de ce type de réduction à Lerdahl et Jackendoff. Mais si le concept est le même, la manière de le définir est quelque peu différente de part et d'autre. Ce que Schenker entendait par prolongations, c'est l'ensemble des patterns que la composition, en se déployant (Auskomponierung), permet d'inclure dans un pattern dominant s'étendant d'un point de départ à un point cadentiel. Dans la théorie de Lerdahl-Jackendoff, on verra que les prolongations sont marquées par les limites des patterns dominants. En fait, quand Schenker parle de prolongation, il désigne une possibilité interne de déploiement, de diversification : il désigne l'intérieur du processus musical ; tandis que Lerdahl et Jackendoff désignent par le même mot les bornes extérieures du processus.

11. "A «preferred" structural description will tend to relate otherwise disparate intuitions and reveal regular structural patterns».

12. A ces deux catégories de règles sont ajoutées quelques règles transformationnelles qui, comme disent les auteurs (p. 11), ne jouent dans la théorie qu'un rôle périphérique: elles concernent l'enjambement, l'élision et la syncopation.

13. "Groups do not receive metrical accent, and beats do not possess any inherent grouping".

14. Des règles de bonne formation et préférentielles se rencontrent à chaque stade de la théorie. J'énumère ici à titre indicatif celles qui concernent la théorie des groupes, particulièrement importantes en psychologie. Elles avaient auparavant fait l'objet d'une étude séparée (Jackendoff-Lerdahl 1981). Pour des raisons d'espace, je ne pourrai poursuivre cette énumération pour les autres composants. Mais j'attire l'attention sur la nécessité d'en avoir la liste sous les yeux si l'on se propose de tester ou de travailler la théorie. L'index complet en est établi à la fin de l'ouvrage (pp. 345 à 352).

15. "The contiguity condition is what makes the slur notation a viable representation of grouping intuitions $»$.

16. "The importance of parallelism in musical structure cannot be overestimated. The more parallelism one can detect, the more internally coherent an analysis becomes, and the less independent information must be processed and retained in hearing or remembering a piece" .

17. On consultera avec profit l'étude minutieuse de l'enjambement et de l'élision (pp. 55 à 62). La place normalement réservée à un compterendu m'impose de renoncer à commenter ce point bien connu de l'analyse qui se rencontre aux points cadentiels.

18. L'arbre prosodique que j'utilise comporte des branches verticales qui indiquent les accents principaux $(T)$. Les branches tirées vers la gauche indiquent les événements de type arsique (A. ATm. TA.), les branches tirées vers la droite indiquent des événements de type désinentiel (K. KTm. TK.). G=groupe. Deux événements ou groupes d'événements de poids égal donnent lieu à des branches consécutives 
respectivement tirées à gauche et à droite d'un noeud central. Ce dernier cas ne se présente pas dans les exemples décrits ici. $\mathrm{A}$. = anacrouse, $\mathrm{T}$. . accent, $\mathrm{K}$. = désinence, $\mathrm{ATm}$. ou KTm. = anacrouse ou désinence sur accent métrique. L'adjonction du $\mathrm{m}$. que je n'utilisais pas précédemment résulte de la distinction entre les espèces d'accents apportée par Lerdahl et Jackendoff. Cette distinction me paraît primordiale et devrait ultérieurement permettre l'effacement du symbole de thesis $(\mathrm{T})$ pour la désignation de l'accent métrique. Je le conserve provisoirement, ne lui attribuant qu'une valeur conventionnelle, Ta. ou TK. = accent arsique ou désinentiel.

19. "Prefer a metrical structure in which a relatively strong beat occurs at the inception of either a) a relatively long pitch-event, b) a relatively long duration of a dynamic, c) a relatively long slur, d) a relatively long pattern of articulation, e) a relatively long duration of a pitch in the relevant levels of the time-span reduction, or $f$ ) a relatively long duration of a harmony in the relevant levels of the time-span reduction (harmonic rhythm)".

20. Cette analyse est accompagnée d'une lecture conçue dans une perspective schenkerienne amendée (Deliège 1982: chap. II) qui comporte deux lignes génératrices. Cette lecture est indépendante de la lecture prosodique, mais contribue à préciser la position des groupes en montrant clairement les patterns harmoniques et contrapuntiques.

21. Une présentation antérieure de cette théorie a paru sous le titre «Toward a Formal Theory of Tonal Music» (1977).

22. "... the listener relates them, more or less unconsciously in the process of listening, to an abstract, simplified structure common to them all".

23. "a. Pitch-events are heard in a strict hierarchy; b. Structurally less important events are not heard simply as insertions, but in a specified relationship to surrounding more important events ".

24. "... is consistent with the methodology of generative linguistics ... The sentences as such in linguistic theorizing are usually taken as given".

25. On trouve au chapitre XI (p. 288) une réponse indirecte à cette interrogation. De fait, les auteurs ne considèrent pas une réduction schenkerienne comme l'équivalent d'une structure profonde, ni la conversion de ce type de réduction en structure de surface comme le produit de règles transformationnelles. Ils notent au sommet de leur argumentation: «Bien qu'une telle approche paraisse initialement plausible, nous la trouvons impraticable musicalement et formellement. Musicalement ... en se confrontant à un passage de quelque complexité et de quelque longueur, il est difficile de spécifier de quoi l'archétype musical est une transformation. Cette difficulté musicale est reflétée formellement dans une difficulté similaire touchant les transformations thématiques : pour dériver différentes structures de surface d'une structure profonde, il faudrait nécessairement admettre une classe de dérivations transfor- 
mationnelles pratiquement dépourvue de contraintes comme possible. Qui plus est, il y a des problèmes pour parvenir à formuler ce que pourraient être les universaux de formes archétypales choisissant la structure profond correcte pour n'importe quelle piéne complexe, et spécifiant systématiquement la dérivation de l'archétype vers la surface musicale pour n'importe quel passage. Pas une, parmi les démarches citées [entendez Schenker et ses successeurs] ne tente sérieusement de résoudre cet ensemble de difficultés formelles".

[ "Although such an approach seems initially plausible, we have found it impracticable both musically and formally. Musically ... in dealing with a passage of any complexity or length it is hard to specify what archetype the music is a transformation of. This musical difficulty is reflected in a formal difficulty similar to the one concerning thematic transformations: in order to derive various surface structures from a deep structure, it would be necessary to admit an almost unconstrained class of permissible transformational derivations. Furthermore, there are the problems of positing a universe of archetypal forms, choosing the correct deep structure for any complex piece, and systematically specifying the derivation from archetype to musical surface for any given passage. None of the approaches cited above makes a serious attempt to solve this collection of formal difficulties ».]

26. Seule une équivoque subsistait touchant la position de la sousdominante que Schenker, naïvement, plaçait à l'extérieur.

27. "It says that particular pitch-events are heard in relation to a particular beat, within a particular group, but it says nothing about how music flows across these segments".

28. "At the smallest levels, metrical structure is the only influence on the choice of time-spans, and the time-spans are regular in length. At some intermediate level, grouping boundaries may interrupt the regularity imposed by the metrical pattern, and the time-spans result from the interaction of meter and grouping. At still larger levels, the timespans are totally determined by grouping structure, and metrical structure is irrelevant ".

29. Je simplifie la citation de la règle en en retirant les symboles d'explicitation. C'est moi qui souligne. Les auteurs ont souligné : « trame temporelle augmentée ", raison d'être de la règle. Cf. l'original anglais : "If a group boundary $G$ intervenes between $B$ and the preceding beat of the same level, $B$ determines an augmented time-span $T_{B}^{\prime}$, which is the interval from $G$ to the end of the regular time-span $T_{B}$ ".

30. "The close dependance of prolongational reduction on timespan reduction constitutes a major claim of our theory. It asserts that the perceived patterns of tension and relaxation in pitch structure depend crucially on the hierarchy of structurally important events within time-spans as defined by meter and grouping. In other words, the listener's understanding of pitch connections in a piece is a function of how he segments his surface". 
31. "Although the trees look similar, they have contrasting interpretations : a) expresses the arc of tonal motion from the piece's structural beginning to its cadence ; b) says first that the piece forms a relaxing prolongation of the tonic and second that the opening I tenses into the $\mathrm{V}$, which in turn relaxes - more strongly than the previous tensing - into the final I».

32. "The choice of events that define prolongational regions is strongly influenced by the relative importance of events in the timespan reduction".

33. «PRPR 1. In choosing the prolongationally most important event $e_{k}$ of a prolongational region $\left(e_{i}-e_{j}\right)$, strongly prefer a choice in which $e_{k}$ is relatively time-span important».

34. "What counts here is the patterns of connection and subordination.... The relative significance of an event is a function of its degree of embedding in the overall structure».

35. "Most theory about contemporary music has been occupied either with the description of compositional systems or with the systematization of analytical procedures not directly related to the listener's understanding. Our approach emphasizes that there is a crucial distinction between the principles by which a piece is composed and the principles by which it is heard (that is, those that permit the listener to construct a mental representation of the piece). As theorists, we are concerned only with the latter $»$.

\section{REFERENCES}

ADORNO, T.W., 1962: Einleitung in die Musiksoziologie. Francfort: Suhrkamp. COOPER, G. et MEYER, $L$.

1960: The Rhythmic Structure of Music. Chicago: University of Chicago Press.

CORNULIER, B. de

1982: Théorie du vers (Rimbaud, Verlaine, Mallarmé). Paris: Seuil. DELIÈGE, $C$.

1974: "Les techniques du rétrospectivisme», International Review of the Aesthetics and Sociology of Music, V/1, 27-42.

1979: "Théories récentes de la tonalité », Degrès, 18, d 1-26 (avec un erratum).

1982a: Les fondements de la musique tonale, une perspective postschenkerienne. Paris : Lattès (à paraître).

1982b: "Some Unsolved Problems in Schenkerian Theory», Colloque sur les grammaires musicales, Université de Modène, octobre 1982, à paraître in Computers and Humanities. 
EPSTEIN, D.

1979: Beyond Orpheus. Cambridge : M.I.T. Press.

FORTE, A. et GILBERT, S.,

1982: Introduction to Schenkerian Theory. New York: Norton.

LERDAHL, F. et JACKENDOFF, R.,

1977: "Toward a Formal Theory of Tonal Music », Journal of Music Theory, XXI/1, 111-172.

1981: "On the Theory of Grouping and Meter», The Musical Quarterly, LXVII/4, 479-506.

1983: A Generative Theory of Tonal Music. Cambridge: M.I.T. Press.

MESSIAEN, $O$.

1944: Technique de mon langage musical. Paris : Leduc.

MEYER, L.B.,

1973: Explaining Music. Chicago: University of Chicago Press. NATTIEZ J.-J.

1975: Fondements d'une sémiologie de la musique. Paris: Union général d'éditions.

1983: "La relation oblique entre le musicologue et le compositeur", Colloque "Le concept de recherche en musique», I.R.C.A.M., Paris, février 1983 (à paraître dans les actes du colloque).

RUWET, N.

1972: Langage, musique, poésie. Paris : Seuil.

SCHENKER, $\mathrm{H}$.

1906: Harmonielehre. Vienne: Universal Edition.

1935: Der Freie Satz. Vienne : Universal Edition. 